\title{
Child Benefit and Fiscal Burden in the Endogenous Fertility Setting
}

\author{
Ryo Ishida \\ Senior Economist, Policy Research Institute, the Ministry of Finance, Japan \\ rrishida112358@gmail.com \\ Kazumasa Oguro \\ Associate Professor, Institute of Economic Research, Hitotsubashi University \\ ZVU07057@nifty.com \\ and \\ Junichiro Takahata \\ Research Associate, JICA Research Institute
}

This version: January 9, 2012

\begin{abstract}
This paper analyzes the possibility of improving the efficiency of child benefit programs in an overlapping generations economy that has endogenous fertility and large government debt levels. We derive the conditions for this improvement using Representative-Consumer and Children-for-Representative-Consumers efficiency criteria in the endogenous fertility setting, as proposed by Michel and Wigniolle (2007). We find that the result crucially depends on the relative amount of accumulated government debt in the economy. When the elasticity of interest rates to child benefit is close to zero and there exists a huge amount of accumulated debt in the economy, financing child benefit programs by issuing debt and using lump-sum tax leads to RC-improvements. This finding is likely to hold in the economies of developed countries that have low fertility rates. We finally provide the implications of these findings on the real economy.
\end{abstract}

Keywords: Endogenous fertility, Pareto-efficiency, child benefit, fiscal burden JEL Classification Numbers: D9, J13, D61

\section{Remark}

The views expressed in this material are those of the authors and do not necessarily reflect the views of JICA, the Ministry of Finance in Japan, or the Policy Research Institute.

\section{Introduction}

The aim of the present paper is to analyze the relationship between child benefit and fiscal burden using an overlapping generations (OLG) model that has endogenous fertility. Lump-sum tax and public debt can be resources for the payment of child benefit. Although the tax burden of each generation focuses on its respective working period, this period also corresponds to the 
Not to be quoted without express written permission from the authors

child-rearing period in some cases. Therefore, implementing child benefit programs financed by lump-sum tax in an exogenous fertility setting is a zero-sum game in that it transfers the fiscal burden to the same generation. Furthermore, financing child benefit programs by issuing public debt is also a zero-sum game in that it transfers the fiscal burden from the current generation to the future generation. However, if certain conditions are satisfied, this benefit has the potential to improve each generation's utility through the mitigation of the per-capita fiscal burden.

In industrialized countries, the fiscal burden has been increasing. In Japan specifically, the debt:GDP ratio is the highest among industrialized countries, even beyond that of Italy. As is well known, the sustainability of the Japanese fiscal and social security system is declining because of its low fertility rate and aging population. This situation has occurred because Japan now holds public debt explicitly and implicitly: explicit debt is approximately 180\% of GDP with regard to government bonds, while implicit debt is approximately $230 \%$ with regard to the social security system, public pensions, medical insurance, and elderly assistance. Therefore, Japan holds approximately 410\% public debt compared with GDP.

Table 1. Public Debt:GDP Ratio and the Total Fertility Rates (TFRs) of Industrialized Countries

\begin{tabular}{|l|c|c|c|c|c|c|}
\hline \multicolumn{1}{|c|}{ Country } & Japan & Italy & France & Germany & UK & US \\
\hline Public debt & 1.72 & 1.17 & 0.71 & 0.69 & 0.46 & 0.61 \\
\hline TFR & 1.33 & 1.32 & 1.87 & 1.28 & 1.66 & 2.04 \\
\hline
\end{tabular}

Source: United Nations (2006), Homepage of the Ministry of Finance, Japan ${ }^{1}$

Moreover, the Baby Boomer generation, which comprises the largest share of the total

1 http://www.mof.go.jp/tax_policy/summary/condition/007.htm 
Not to be quoted without express written permission from the authors

population, is beginning to cross over to the benefit side of the social security system. Thus, attempts to reduce benefits will face political limitations, thereby implying that Japan's massive public debt must be paid for by the current working generation and future generations.

In addition, the fertility rate in Japan has been decreasing since the 1950s. In order to maintain the current population level of 128 million $^{2}$, it is considered necessary for each Japanese woman to bear 2.08 children. This TFR in Japan was above 2.08 before the 1970s, but since then, it has fallen below that number ${ }^{3}$. The relationships between (explicit) debt:GDP ratio and fertility rate in developed countries are shown in Table 1.

These demographic factors raise the following question: what is the most economically efficient way for this burden to be shared by each generation? The answer will essentially differ depending on whether the model is based on exogenous fertility or endogenous fertility.

For this reason, recent studies clarify that the Pareto-efficiency condition of the exogenous fertility model differs from that of the endogenous one. First, in the case of an exogenous fertility model, we make use of the OLG model that was introduced by Diamond (1965). Three types of steady states exist in this model: under-accumulation, golden rule, and over-accumulation. The first two steady states are Pareto-efficient, but the third is not. In addition, the empirical study by Abel et al. (1989) reports that in industrialized countries dynamic efficiency is satisfied. In a steady state, dynamic efficiency corresponds to under-accumulation (or golden rule). Therefore, the possibility that industrialized countries are in a state of under-accumulation seems high.

In an exogenous fertility setting, an allocation is said to be Pareto-efficient if it is impossible to make certain individuals better off without making other individuals worse off. For this reason,

2 Based on Census 2010

${ }^{3}$ Latest TFR in Japan is 1.39 in 2010 (Vital Statistics of Japan by the Ministry of Health, Labour and Welfare) 
Not to be quoted without express written permission from the authors

in an exogenous case, we cannot improve any generation's utility while at the same time sacrificing another’s utility.

However, recent studies clarify the properties of the competitive equilibrium in an endogenous fertility setting. Raut and Srinivasan (1994) and Chakrabarti (1999) analyze the properties of the inter-temporal equilibrium in such a setting. Moreover, Conde-Ruiz and Gimenez (2002) and Golosov et al. (2004) define Pareto-efficiency criteria in an endogenous fertility framework.

Building on these studies, Michel and Wigniolle $(2007)^{4}$ point out the possibility that under-accumulation may not be efficient in an endogenous fertility setting. This implies that certain policies may improve one generation's welfare without making another worse off, even when it is in an under-accumulation state near the steady state. Moreover, Michel and Wigniolle (2007) confirm that the Representative-Consumer efficient (RC-efficient ${ }^{5}$ ) condition, which is a concept developed in their study, is deeply connected with the sign-of-inequality relationship between child-rearing cost and wage rate. In other words, if certain policies influence this relationship, they may be able to improve RC-efficiency.

Michel and Wigniolle (2007) also prove that using an OLG model that has endogenous population growth might improve RC-efficiency in the case of a state of under-accumulation. However, they do not analyze an economy model using public debt. Therefore, there is great research interest in the possibility of improving RC-efficiency in an economy that has huge

\footnotetext{
4 Although several approaches have endogenized fertility decisions, Michel and Wigniolle (2007) depend on the benchmark framework, which assumes that children are consumption goods that appear in the utility function of their parents (Becker, 1960; Willis, 1973; Eckstein and Wolpin, 1985). Other approaches are based on the additional assumption of descendant altruism, as in Becker and Barro (1988) or the assumption of ascendant altruism and the strategic behavior of parents, as in Nishimura and Zhang (1992).

5 This can be defined as an allocation for which no other allocation exists that would lead to a higher level of utility for all generations with a strict improvement for (at least) one generation.
} 
Not to be quoted without express written permission from the authors

public debt, a low fertility rate, and endogenous population growth such as that of Japan.

In this paper, we thus focus on child benefit programs that are financed by public debt, which influence the conditions necessary for RC-efficiency in the following way. First, there exists a path from reducing the per-capita fiscal burden to increasing the fertility rate, which can be found in simple models that do not include capital accumulation. Second, as shown by models that include capital accumulation, child benefit may affect an individual's expenditure through current fertility level and interest rates, which cause the consumption amount in the subsequent period. The first condition has a particular effect when an economy holds huge levels of public debt such as that of Japan.

Intuitively, there is the possibility to improve RC-efficiency ${ }^{6}$ by financing a child benefit program with newly issued debt when the accumulated amount of public debt is huge based on the following logic. Suppose such a child benefit program raises the fertility rate to a certain level. The influence of this newly issued debt on accumulated debt levels depends on the size of the latter. If the effect of the rise in fertility is the same irrespective of the level of accumulated debt, then such a policy may lessen per-capita debt without harming any generation. In this scenario, even the initial generation is not made worse off because they do not endure the burden. However, the situation of the initial generation might worsen if the child benefit program were to be financed by lump-sum tax. We first show this situation using a simple model and then derive conditions in a general setting. Note that, with this logic, this approach achieves not only RC-improvement but also Children-for-Representative-Consumers (CRC)-improvement ${ }^{7}$.

This remainder of this paper is organized as follows. In Section 2, we introduce a simple

\footnotetext{
6 A change to a different allocation that makes (at least) one generation better off without making any other generation worse off.

7 CRC-improvement means RC-improvement that does not decrease any generation's fertility rate.
} 
Not to be quoted without express written permission from the authors

model for grasping an intuitive understanding. In Section 3, we use this model for our main analysis. In Section 4, we derive the conditions for RC-improvement using the model and describe the additional conditions for CRC-improvement. In Section 5, we analyze the superiority of public debt and tax with regard to financing child benefit programs. Section 6 concludes.

\section{Simple analysis}

In this section, we analyze a simple model to show the characteristics of child benefit programs that are financed by public debt in preparation for assessing the rigorous model in Section 3. As an example, we first intuitively analyze the relationship between child benefit and fiscal burden in the case of intergeneration selfishness in a simple economy that has only two generations, namely a parent (first) generation and a child (second) generation. Second, using an OLG model that features these two generations, we show that child benefit can improve RC-efficiency.

For simplicity, we consider that individuals live two periods, namely young and old, and that they have children when they are young. We assume that the second generation does not have children and that government expenditure is set to zero in the baseline case. The debt level at the beginning is set to $D$ and the government subsidizes $\delta$ per child for child-rearing activity, which is financed by issuing bonds. In this simple model, we let $N$ denote the population of the first generation, $n N$ that of the second generation, interest rates, $z$ child-rearing costs, $X_{j}$ and $Y_{j}$ consumption when young and old, $W$ lifetime income, and $T_{j}(j=1,2)$ the fiscal burden in lump-sum tax. Using the variables above, we find the following budget constraints for 
Not to be quoted without express written permission from the authors

a representative household:

$$
\begin{gathered}
(z-\delta) n+X_{1}+\frac{Y_{1}}{1+r}=W_{1}-T_{1} \\
X_{2}+\frac{Y_{2}}{1+r}=W_{2}-T_{2}
\end{gathered}
$$

The intertemporal government budget constraints are:

$$
D+\delta n N=T_{1} N+\frac{T_{2}}{1+r} n N
$$

By solving the per-capita fiscal burden of the second generation from equations (1) to (3), we get the following relationship:

$$
T_{2}=(1+r) \frac{D+\delta n N-T_{1} N}{n N}
$$

If $\partial T_{2} / \partial \delta<0$ is satisfied, enlarging child benefit programs financed by bonds decreases the fiscal burden of the second generation without decreasing any generation's fertility rate. It is thus possible to rewrite the condition as:

$$
\frac{d}{n}>\frac{\delta}{\eta_{\delta n}} \quad \text { where } \quad d \equiv D / N-T_{1} \quad \text { and } \quad \eta_{\delta n} \equiv \frac{\delta}{n} \frac{\partial n}{\partial \delta}
$$

The left-hand side represents the fiscal burden of the second generation, whereas the denominator on the right-hand side represents the elasticity of fertility to child benefit programs. As long as the ratio of child-rearing subsidy to elasticity is less than the per-capita fiscal burden of the second generation, child benefit programs decrease the per-capita fiscal burden of the second generation. Specifically, the after-tax lifetime income of the second generation increases, which implies that lifetime utility rises. The lifetime utility of the first generation also rises because of the child benefit programs financed by bonds. In addition, the fertility rate rises, too. Overall, in the case that equation (5) holds, a child-rearing policy that is financed by bonds may 
Not to be quoted without express written permission from the authors

attain not only RC-improvement but also CRC-improvement.

\section{Model}

In this section, we construct a model to consider the condition of financing child benefit by bonds in order to affect RC-improvement and, possibly, CRC-improvement. Detailed settings are shown in the following subsections.

\subsection{Household}

Generation $t$ live across two periods, namely period $t$ when they are young and period $t+1$ when they are old. Furthermore, they earn a lifetime income, enjoy consumption $X_{t}$ when young and $Y_{t+1}$ when old, and raise children $n_{t}$ at cost $z$, subsidized by $\delta_{t}$. Generation $t$ have to take over per-capita debt $d_{t}$ from generation $t-1$ by paying lump-sum tax $T_{t}$ when young, and they give their per-capita debt $d_{t+1}$ to the following generation $t+1$ when old.

Assumption $1 U$ is a function of $\mathrm{R}_{+}^{3}$ to $\mathrm{R} \cup\{-\infty\}$, and $U$ maps $\mathrm{R}_{++}^{3}$ to $\mathrm{R}$, with

$$
U(\bar{n}, \bar{X}, \bar{Y})=\lim _{(n, X, Y) \rightarrow(\bar{n}, \bar{X}, \bar{Y})} U(n, X, Y) \quad \text { for every }(\bar{n}, \bar{X}, \bar{Y}) \in \mathrm{R}_{+}^{3} / \mathrm{R}_{++}^{3}
$$

$U$ is twice continuously differentiable on $\mathrm{R}_{++}^{3}$, strictly concave, increasing in each argument, homogeneous of degree one, and satisfies the Inada conditions:

$$
\lim _{n \rightarrow 0} U_{n}^{\prime}=\lim _{X \rightarrow 0} U_{X}^{\prime}=\lim _{Y \rightarrow 0} U_{Y}^{\prime}=+\infty
$$

In this case, the lifetime utility and budget constraints of generation $t$ are described as 
follows:

$$
\begin{gathered}
U_{t}=U\left(n_{t}, X_{t}, Y_{t+1}\right) \\
\left(z-\delta_{t}\right) n_{t}+X_{t}+S_{t}=W_{t}-T_{t} \\
Y_{t+1}=R_{t+1} S_{t}
\end{gathered}
$$

The first-order conditions for maximizing lifetime utility are as follows:

$$
U_{X}=R_{t+1} U_{Y}=\frac{U_{n}}{Z-\delta_{t}}
$$

From the above equations, we can derive the following relationships:

$$
\begin{gathered}
X_{t}=X\left(W_{t}-T_{t}, R_{t+1}, \delta_{t}\right) \\
Y_{t+1}=Y\left(W_{t}-T_{t}, R_{t+1}, \delta_{t}\right) \\
n_{t}=n\left(W_{t}-T_{t}, R_{t+1}, \delta_{t}\right) \\
S_{t}=S\left(W_{t}-T_{t}, R_{t+1}, \delta_{t}\right)
\end{gathered}
$$

Functions $X, Y, n$, and $s$ are defined on $\mathrm{R}_{++}^{3}$ and are continuously differentiable.

\subsection{Firm}

We assume that, in period $t$, there exists a representative firm that produces goods using capital $K_{t}$ and labor $L_{t}$ under perfect competition using the following function, which is homogeneous of degree one. We define $f$ as $f(k) \equiv F(k, 1)$.

$$
Q_{t}=F\left(K_{t}, L_{t}\right)
$$

Assumption $2 f: \mathrm{R}_{+} \rightarrow \mathrm{R}_{+}$, and for all $k>0, f^{\prime}(k)>0$ and $f^{\prime \prime}(k)<0$. 
Then, we get the following condition from profit maximization:

$$
\begin{gathered}
W_{t}=f\left(\frac{K_{t}}{L_{t}}\right)-\frac{K_{t}}{L_{t}} f^{\prime}\left(\frac{K_{t}}{L_{t}}\right) \equiv W\left(\frac{K_{t}}{L_{t}}\right) \\
R_{t}=f^{\prime}\left(\frac{K_{t}}{L_{t}}\right) \equiv R\left(\frac{K_{t}}{L_{t}}\right)
\end{gathered}
$$

\subsection{Government}

Suppose that the population of generation $t$ is expressed as $N_{t}=n_{t-1} N_{t-1}$, and that the government subsidizes child-rearing under the following budget constraints. The reimbursement of the per-capita debt of generation $t-1$ and child-rearing subsidy $\delta_{t}$ are financed by lump-sum tax $T_{t}$ and newly issued bonds $d_{t}$ :

$$
\begin{aligned}
& T_{t} N_{t}+d_{t} N_{t}=R_{t} d_{t-1} N_{t-1}+n_{t} \delta_{t} N_{t} \\
\Leftrightarrow & T_{t}=\frac{R_{t} d_{t-1}}{n_{t-1}}+n_{t} \delta_{t}-d_{t}
\end{aligned}
$$

\subsection{Market equilibrium}

Suppose that the labor market is balanced as $L_{t}=N_{t}$, and that the capital and savings in the capital market are balanced. Then, with $k_{t} \equiv K_{t} / N_{t}$, we have the following:

$$
\begin{gathered}
W_{t}=W\left(k_{t}\right) \quad \text { and } \quad R_{t}=R\left(k_{t}\right) \\
K_{t+1}=N_{t}\left(S_{t}-d_{t}\right)
\end{gathered}
$$

Because $R\left(k_{t}\right)$ is a strictly decreasing function of $k_{t}$ because of $f^{\prime \prime}(k)<0$, this function is 
Not to be quoted without express written permission from the authors

bijective. Thus, we have the following strictly decreasing function:

$$
k_{t}=k\left(R_{t}\right)
$$

Equation (19) is verified as being equivalent to the following commodity market-clearing condition by a simple operation:

$$
f\left(k_{t}\right)=n_{t} k_{t+1}+X_{t}+\frac{Y_{t}}{n_{t-1}}+n_{t} Z
$$

Definition 1 Starting from initial conditions $N_{-1}, N_{0}, K_{0}$, and $Y_{0}=R_{0}\left(d_{-1}+\left(K_{0} / N_{-1}\right)\right)$, given debt management policies and child-rearing subsidies $\left\{\left(d_{t}, \delta_{t}\right)_{t=0}^{\infty}\right\}$, an inter-temporal equilibrium is a sequence $\left\{\left(K_{t}, N_{t}, X_{t}, Y_{t}, n_{t}\right)_{t=0}^{\infty}\right\}$, which satisfies (7)-(9) and (17)-(19).

\section{The inter-temporal equilibrium}

In this section, based on the model presented in Section 3, we examine the condition for financing child benefit by issuing bonds in order to achieve RC-improvement. Then, an example using a simple function is considered.

First, we derive the condition of child benefit programs that improves the lifetime utility levels of all generations without sacrificing the welfare of any single generation. Because it is difficult to derive such a condition rigorously in an analytical sense, for simplicity, we thus assume that $U=U_{t}(\cdot)$ is homogeneous of degree one. In addition, we define the variables as follows:

$$
\tilde{n}_{t} \equiv \frac{n_{t}}{W_{t}-T_{t}}
$$


Not to be quoted without express written permission from the authors

$$
\begin{gathered}
\tilde{X}_{t} \equiv \frac{X_{t}}{W_{t}-T_{t}} \\
\tilde{Y}_{t+1} \equiv \frac{Y_{t+1}}{W_{t}-T_{t}} \\
\tilde{S}_{t} \equiv \frac{S_{t}}{W_{t}-T_{t}} \\
\tilde{d}_{t} \equiv \frac{d_{t}}{W_{t}-T_{t}}-\tilde{n}_{t} \delta_{t} . \\
\tilde{U}_{t} \equiv \frac{U_{t}}{W_{t}-T_{t}} .
\end{gathered}
$$

The government budget constraints can be rewritten as:

$$
T_{t}=\frac{\frac{R_{t} \tilde{d}_{t-1}}{\tilde{n}_{t-1}}+R_{t} \delta_{t-1}-W_{t} \tilde{d}_{t}}{1-\tilde{d}_{t}}
$$

Moreover, the budget constraints (7) and (8) can be transformed as follows:

$$
\left(z-\delta_{t}\right) \tilde{n}_{t}+\tilde{X}_{t}+\frac{\tilde{Y}_{t+1}}{R_{t+1}}=1
$$

From equations (9) and (22), we can solve the variables of equation (21) as a function of $\left(z-\delta_{t}, R_{t+1}\right)$. Substituting these variables into (19), we obtain the following:

$$
\begin{gathered}
\tilde{n}_{t}\left(z-\delta_{t}, R_{t+1}\right)\left(k_{t+1}+\delta_{t}\right)=\tilde{S}_{t}\left(z-\delta_{t}, R_{t+1}\right)-\tilde{d}_{t} \\
\Leftrightarrow k_{t+1}=k_{t+1}\left(\delta_{t}\right)
\end{gathered}
$$

Proposition 1 Given $k_{0}=K_{0} / N_{0}$ and $\left\{\left(\tilde{d}_{t}\right)_{t=0}^{\infty}\right\}$, an inter-temporal equilibrium is characterized by the sequence $\left\{\left(\delta_{t}\right)_{t=0}^{\infty}\right\}$ such that $\forall t \geq 0$,

$$
\tilde{n}_{t}\left[z-\delta_{t}, R\left(k_{t+1}\right)\right]\left(k_{t+1}+\delta_{t}\right)=\tilde{S}_{t}\left[z-\delta_{t}, R\left(k_{t+1}\right)\right]-\tilde{d}_{t}
$$


Not to be quoted without express written permission from the authors

The proof is straightforward. A sequence $\left(k_{t}\right)_{t \geq 0}$ is characterized by a sequence $\left(\delta_{t}\right)_{t \geq 0}$ in this setting, while an inter-temporal equilibrium is characterized by a sequence $\left(k_{t}\right)_{t \geq 0}$ according to Michel and Wigniolle (2007). Hence, an inter-temporal equilibrium is characterized by a sequence $\left(\delta_{t}\right)_{t \geq 0}$.

We define the function $\Gamma$ as:

$$
\Gamma(z-\delta, k) \equiv \tilde{n}\left[z-\delta, f^{\prime}(k)\right](k+\delta)-\tilde{s}\left[z-\delta, f^{\prime}(k)\right]+\tilde{d}
$$

$\Gamma$ is defined on $R_{++}^{2}$ and is continuously differentiable. Equation (24) can be rewritten as:

$$
\Gamma\left(z-\delta_{t}, k_{t+1}\left(\delta_{t}\right)\right)=0
$$

In this setting, the equation is no longer dynamic because the function is only of $k_{t+1}$ but not of $k_{t}$. Because $k_{t+1}$ is a function of $\delta_{t}$, once we have a sequence of $\left(\delta_{t}\right)_{t \geq 0}$, a unique inter-temporal equilibrium $\left(\delta_{t}\right)_{t \geq 0}$ may exist, as described next. Before that, we need to make some assumptions on how savings and the fertility rate react to changes in interest rates and child benefit.

In the following, we deal with several cases in terms of preferences. In the first case, we consider the case with preferences with which $\partial k_{t+1} / \partial \delta_{t}>0$ is satisfied. Next, we consider the opposite case. Then, we show that, under certain assumptions, $\partial k_{t+1} / \partial \delta_{t}>0$ will not occur. Before that, we make the following assumption.

Assumption 3-1 Following a change in interest rates, we assume that savings and the fertility 
rate change as follows:

$$
\begin{gathered}
\tilde{s}_{R} \geq 0, \eta_{R \tilde{n}}<\eta_{R k} \frac{k(R)}{k(R)+\delta} \text { where } \\
\eta_{R \tilde{n}} \equiv \frac{R_{t+1}}{\tilde{n}_{t}} \frac{\partial \tilde{n}_{t}}{\partial R_{t+1}}, \eta_{R k} \equiv-\frac{R_{t+1}}{k_{t+1}} \frac{\partial k_{t+1}}{\partial R_{t+1}}=-\frac{R_{t+1}}{k\left(R_{t+1}\right)} \frac{\partial k\left(R_{t+1}\right)}{\partial R_{t+1}}
\end{gathered}
$$

The first part of the above assumption means that savings as a proportion of after-tax income increase when interest rates increase. The second part of it means that the elasticity of the adjusted fertility rate with respect to interest rates is negative or, even if it is positive, it is inelastic compared with asset elasticity. Note that as $\partial k\left(R_{t+1}\right) / \partial R_{t+1}<0 \Leftrightarrow \eta_{R k}>0$ holds from $f^{\prime \prime}(k)<0$, the second part of the above assumption is satisfied if $\eta_{R \tilde{n}} \leq 0$ holds. Thus, the following assumption is sufficient under which assumption 3-1 holds.

Assumption 3-2 The following assumption is the sufficient condition of assumption 3-1:

$$
\tilde{s}_{R} \geq 0, \tilde{n}_{R} \leq 0
$$

Proposition 2 Under assumptions 1, 2, and 3-1, $\forall k_{0}>0$; for any sequence $\forall\left\{\delta_{t}\right\}_{t=0}^{\infty}$, there exists a unique inter-temporal equilibrium $\left\{k_{t+1}\right\}_{t=0}^{\infty}$ starting from a given initial condition $k_{0}>0$.

Proof See Appendix A.

The difference from the findings of Michel and Wigniolle (2007) is that there are no 
Not to be quoted without express written permission from the authors

dynamics in $k$ in our model because we have assumed homogeneity with household preferences, which drops the effect of wage rate determined by the capital level in the same period.

Our next interest is in the relationship between $k$ and $\delta$. In the following subsection, we consider two cases about this sign: one is the case when $\partial k / \partial \delta>0$ and the other is when $\partial k / \partial \delta<0$. For this, we need to make additional assumptions about $s$ and $n$ given a change in $\delta$.

Definition 2 An inter-temporal equilibrium $\left(K_{t}, N_{t}, X_{t}, Y_{t}, n_{t}\right)_{t \geq 0}$ is said to be converging if the sequence $k_{t}=K_{t} / N_{t}$ converges to a limit $\bar{k}>0$ when approaches infinity. If $k_{t}$ converges to a limit $\bar{k}$, it is straightforward to show that $R_{t}=R\left(k_{t}\right), W_{t}=W\left(k_{t}\right), X_{t}, Y_{t}$, and are converging to constant values $\bar{R}, \bar{W}, \bar{X}, \bar{Y}$, and $\bar{n}$.

Definition 3 A converging inter-temporal equilibrium $\left(K_{t}, N_{t}, X_{t}, Y_{t}, n_{t}\right)_{t \geq 0}$ is said to converge in a state of under-accumulation if $\bar{R}>\bar{n}$. It is said to converge in a state of over-accumulation if $\bar{R}<\bar{n}$

Definition 4 (RC-allocation) A feasible allocation with representative consumers (or RC-allocation) is a sequence $\left(K_{t}^{i}, N_{t}^{i}, X_{t}^{i}, Y_{t}^{i}, n_{t}^{i}\right)_{t \geq 0}$ of positive variables that satisfies $\forall t \geq 0$ :

$$
\begin{gathered}
F\left(K_{t}, N_{t}\right)=K_{t+1}+N_{t} X_{t}+N_{t-1} Y_{t}+N_{t+1} Z \\
N_{t+1}=n_{t} N_{t} .
\end{gathered}
$$


Definition 5 (RC-dominance) Let $\left(K_{t}^{i}, N_{t}^{i}, X_{t}^{i}, Y_{t}^{i}, n_{t}^{i}\right)_{t \geq 0}$ for $i=1,2$ be two feasible RC-allocations. Allocation 1 is said to RC-dominate allocation 2 if it leads to a higher level of utility for all generations, with a strict improvement for (at least) one generation. Formally, this is:

$$
\begin{gathered}
\forall t \geq 0, U\left(X_{t}^{1}, Y_{t+1}^{1}, n_{t}^{1}\right) \geq U\left(X_{t}^{2}, Y_{t+1}^{2}, n_{t}^{2}\right), \\
\exists t_{0} \geq 0, \quad \text { such that } U\left(X_{t 0}^{1}, Y_{t 0+1}^{1}, n_{t 0}^{1}\right)>U\left(X_{t 0}^{2}, Y_{t 0+1}^{2}, n_{t 0}^{2}\right) .
\end{gathered}
$$

Definition 6 (CRC-dominance) Let $\left(K_{t}^{i}, N_{t}^{i}, X_{t}^{i}, Y_{t}^{i}, n_{t}^{i}\right)_{t \geq 0}$ for $i=1,2$ be two feasible RC-allocations. Allocation 1 is said to CRC-dominate allocation 2 if it leads to a higher level of utility for all generations, with a strict improvement for (at least) one generation, without decreasing any generation’s fertility rate. Formally, this is:

$$
\begin{gathered}
\forall t \geq 0, U\left(X_{t}^{1}, Y_{t+1}^{1}, n_{t}^{1}\right) \geq U\left(X_{t}^{2}, Y_{t+1}^{2}, n_{t}^{2}\right), \\
\forall t \geq 0, n_{t}^{1} \geq n_{t}^{2} \\
\exists t_{0} \geq 0, \quad \text { such that } U\left(X_{t 0}^{1}, Y_{t 0+1}^{1}, n_{t 0}^{1}\right)>U\left(X_{t 0}^{2}, Y_{t 0+1}^{2}, n_{t 0}^{2}\right) .
\end{gathered}
$$

Definition 7 (RC-improvement) Let $\left(K_{t}^{i}, N_{t}^{i}, X_{t}^{i}, Y_{t}^{i}, n_{t}^{i}\right)_{t \geq 0}$ for $i=1,2$ be two feasible RC-allocations. If allocation 1 RC-dominates allocation 2, a shift from allocation 2 to allocation 1 is termed an RC-improvement.

Definition 8 (CRC-improvement) Let $\left(K_{t}^{i}, N_{t}^{i}, X_{t}^{i}, Y_{t}^{i}, n_{t}^{i}\right)_{t \geq 0}$ for $i=1,2$ be two feasible RC-allocations. If allocation 1 CRC-dominates allocation 2, a shift from allocation 2 to 
allocation 1 is termed a CRC-improvement.

If allocation 2 were changed to allocation 1 using child benefit programs, an RC-improvement or CRC-improvement would be achieved.

4.1. Case 1: if $\eta_{\dot{\delta}}>\eta_{\check{\delta}}$ and $\eta_{\delta s}>\frac{\delta_{t} \tilde{n}_{t}}{\tilde{d}_{t}}$ where

$$
\eta_{\delta s} \equiv \frac{\delta_{t}}{\tilde{s}_{t}} \frac{\partial \tilde{s}_{t}}{\partial \delta_{t}}, \eta_{\delta n} \equiv \frac{\delta_{t}}{\tilde{n}_{t}} \frac{\partial \tilde{n}_{t}}{\partial \delta_{t}}
$$

First, we consider the sign of $\partial k_{t+1} / \partial \delta_{t}$. Taking the derivative of equation (23) with respect to $\delta_{t}$, we have:

$$
\begin{gathered}
\frac{\partial \tilde{n}_{t}}{\partial \delta_{t}}\left(k_{t+1}+\delta_{t}\right)+\frac{\partial \tilde{n}_{t}}{\partial R_{t+1}} \frac{\partial R_{t+1}}{\partial \delta_{t}}\left(k_{t+1}+\delta_{t}\right)+\tilde{n}_{t}\left(\frac{\partial \tilde{k}_{t+1}}{\partial \delta_{t}}+1\right)=\frac{\partial \tilde{S}_{t}}{\partial \delta_{t}}+\frac{\partial \tilde{S}_{t}}{\partial R_{t+1}} \frac{\partial R_{t+1}}{\partial \delta_{t}} \\
\Leftrightarrow \frac{\partial k_{t+1}}{\partial \delta_{t}}=\frac{\frac{\partial \tilde{S}_{t}}{\partial \delta_{t}}-\frac{\partial \tilde{n}_{t}}{\partial \delta_{t}}\left(k_{t+1}+\delta_{t}\right)-\tilde{n}_{t}}{\left(\tilde{n}_{t}+\frac{\partial \tilde{n}_{t}}{\partial R_{t+1}} \frac{\partial R_{t+1}}{\partial k_{t+1}}\left(k_{t+1}+\delta_{t}\right)-\frac{\partial \tilde{S}_{t}}{\partial R_{t+1}} \frac{\partial R_{t+1}}{\partial k_{t+1}}\right)}=\frac{\eta_{\delta s} \frac{\tilde{S}_{t}}{\delta_{t}}-\eta_{\delta n} \frac{\tilde{n}_{t}}{\delta_{t}}\left(k_{t+1}+\delta_{t}\right)-\tilde{n}_{t}}{\left(\tilde{n}_{t}+\frac{\partial \tilde{n}_{t}}{\partial R_{t+1}} \frac{\partial R_{t+1}}{\partial k_{t+1}}\left(k_{t+1}+\delta_{t}\right)-\frac{\partial \tilde{S}_{t}}{\partial R_{t+1}} \frac{\partial R_{t+1}}{\partial k_{t+1}}\right)} \\
=\frac{\eta_{\delta s} \frac{\tilde{n}_{t}\left(k_{t+1}+\delta_{t}\right)+\tilde{d}_{t}}{\delta_{t}}-\eta_{\delta n} \frac{\tilde{n}_{t}\left(k_{t+1}+\delta_{t}\right)}{\delta_{t}}-\tilde{n}_{t}}{\left(\tilde{n}_{t}+\frac{\partial \tilde{n}_{t}}{\partial R_{t+1}} \frac{\partial R_{t+1}}{\partial k_{t+1}}\left(k_{t+1}+\tilde{n}_{t}\right)+\frac{\partial \tilde{S}_{t}}{\partial R_{t+1}} \frac{\partial R_{t+1}}{\partial k_{t+1}}\right)}=\frac{\left.\tilde{n}_{\delta s}-\eta_{\delta n}\right) \frac{\left.\tilde{n}_{t}+\delta_{t+1}\right)}{\delta_{t}}}{\left(\tilde{n}_{t}+\frac{\partial \tilde{n}_{t}}{\partial R_{t+1}} \frac{\partial R_{t+1}}{\partial k_{t+1}}\left(k_{t+1}+\delta_{t}\right)-\frac{\partial \tilde{S}_{t}}{\partial R_{t+1}} \frac{\partial R_{t+1}}{\partial k_{t+1}}\right)}>0
\end{gathered}
$$

Provided assumption 3-1 holds, we derive the following proposition: 
Proposition 3 RC-improvement is achieved by financing child benefit using public debt when the following is true:

$$
\begin{gathered}
\delta_{t} n_{t}>\eta_{\delta R} \frac{Y_{t+1}}{R_{t+1}} \\
\eta_{\delta n}>\frac{n_{t} \delta_{t}}{d_{t}}
\end{gathered}
$$

where

$$
\eta_{\delta n} \equiv \frac{\delta_{t}}{n_{t}} \frac{\partial n_{t}}{\partial \delta_{t}}, \eta_{\delta R} \equiv-\frac{\delta_{t}}{R_{t+1}} \frac{\partial R_{t+1}}{\partial \delta_{t}}
$$

Proof See Appendix B.

The first condition implies that the income from child benefit programs must exceed the loss of consumption in the second period because of a decrease in interest rates. If individuals originally plan to consume more in the second period, an interest rate decrease might make them worse off because of negative income effects. However, if individuals have children, child benefit may bring positive income effects. In order to satisfy this condition, it is necessary for the second effect to dominate the first. If the elasticity is small enough, the condition is satisfied as long as there exists a certain level of child benefit program available.

The second condition requires that the elasticity of fertility rate to child benefit be bigger than is the ratio of per-capita amount of child benefit to that of accumulated debt. In countries that have huge levels of accumulated debt but relatively small levels of child benefit, the second condition is likely to hold; however, this requires that child benefit programs be bigger for the 
Not to be quoted without express written permission from the authors

first condition to be true.

It is interesting that equations (5) and (27) are the same. This implies that condition (27) more likely holds as the amount of per-capita debt grows, which is true for the financial situation of the government sector in Japan, as examined in Section 2. However, in the model that has capital accumulation presented in this section, we need the additional condition (26). It would then be possible to examine the possibility of RC-improvement by granting child-rearing subsidies financed by bonds if we could confirm that equations (26) and (27) actually hold in an empirical analysis. An interesting point is whether it is possible to implement RC-improvement in the real economy.

We briefly discuss that possibility in the following subsection by focusing on the case of the Japanese economy, which, as previously mentioned, has a huge level of per-capita debt and a low fertility rate. From equations (26) and (27), we can derive the condition of $\delta_{t}$ as follows:

$$
\frac{\eta_{\delta \delta} d_{t}}{n_{t}}>\delta_{t}>\eta_{\delta R} \frac{Y_{t+1}}{R_{t+1} n_{t}}
$$

Although it is hard to estimate the actual elasticities of the Japanese economy, $\eta_{\partial R}$ is not considered too high (it might even be close to zero). The Japanese economy is large enough that interest rates remain almost unaffected by such a policy. By contrast, $\eta_{\dot{\delta}}$ is assumed to be 0.05 , which implies that a $100 \%$ increase in child benefit would increase the fertility rate by $10 \%$. In this case, when child benefit increases from 10,000 yen to 20,000 yen every month, the fertility rate might increase from 1.34 to 1.39 . If the debt amount $d_{t}$ is 20 million yen, then as long as child benefit per child is below 1 million yen, this condition is satisfied. Furthermore, although the Japanese population is not decreasing dramatically at present, it is expected to decline much more rapidly in the future. In such a case, per-capita debt would increase dramatically, thereby 
leading to a greater possibility for RC-improvement.

4.2. Case 2: if $\tilde{s}_{\delta} \leq 0$ and $\tilde{n}_{\delta}>0$ are satisfied

First, we consider the sign of $\partial k_{t+1} / \partial \delta_{t}$.

$$
\frac{\partial k_{t+1}}{\partial \delta_{t}}=\frac{\frac{\partial \tilde{S}_{t}}{\partial \delta_{t}}-\frac{\partial \tilde{n}_{t}}{\partial \delta_{t}}\left(k_{t+1}+\delta_{t}\right)-\tilde{n}_{t}}{\left(\tilde{n}_{t}+\frac{\partial \tilde{n}_{t}}{\partial R_{t+1}} \frac{\partial R_{t+1}}{\partial k_{t+1}} k_{t+1}-\frac{\partial \tilde{S}_{t}}{\partial R_{t+1}} \frac{\partial R_{t+1}}{\partial k_{t+1}}\right)}<0
$$

In this case, we need the conditions for improving efficiency as discussed above.

Proposition 4 RC-improvement is achieved by financing child benefit using public debt when the following is true:

$$
\eta_{\delta n}>\varepsilon_{\delta R}-\eta_{\delta W} \frac{W_{t} \tilde{n}_{t-1}}{R_{t} \tilde{d}_{t-1}}+\left(1+\varepsilon_{\delta R}\right) \frac{\tilde{n}_{t-1} \delta_{t-1}}{\tilde{d}_{t-1}}
$$

where

$$
\varepsilon_{\delta R} \equiv \frac{\delta_{t}}{R_{t+1}} \frac{\partial R_{t+1}}{\partial \delta_{t}}, \quad \eta_{a v} \equiv \frac{\delta_{t}}{W_{t+1}} \frac{\partial W_{t+1}}{\partial \delta_{t}}
$$

This proposition is true even in the case of $\partial k_{t+1} / \partial \delta_{t} \leq 0$.

Proof See Appendix C.

This condition implies that the elasticity of the fertility rate to child benefit should be high 
Not to be quoted without express written permission from the authors

enough to dominate the right-hand side effects. We consider a small change of $\delta_{t}$. First, when the elasticity of $k$ to $\delta$ is considered to be not too high, it is likely that the condition is satisfied. In that case, both $\left|\varepsilon_{\delta R}\right|$ and $\left|\eta_{\delta w}\right|$ are close to zero, which also renders the right-hand side of equation (28) close to zero and thus satisfies the condition.

Second, as $d_{t-1}$ increases, the condition becomes more likely to be satisfied, as shown in Proposition 3. This shows that the possibility of improving efficiency by financing child benefit is higher in both cases when the level of existing debt is huge. We provide sufficient conditions for RC-improvement in the case of $\partial k_{t+1} / \partial \delta_{t}>0$ and $\partial k_{t+1} / \partial \delta_{t}<0$. By using the Cobb-Douglas utility function and the production function $f\left(k_{t}\right)=A_{t} k_{t}^{\rho}$, the relationship between $k$ and $\delta$ seems to hold: $\partial k_{t+1} / \partial \delta_{t}<0$. This characteristic is expanded as follows:

Proposition 5 Under Assumptions 1, 2, and 3-2, $\partial k_{t+1} / \partial \delta_{t} \leq 0$ holds.

Proof See Appendix D.

We next provide an example of when RC-improvement is achieved. This example satisfies assumptions 1, 2, and 3-2, and thus, Proposition 4 is valid in this example.

Example 1 We assume specific forms for preferences and production technology in the above model. Suppose we have preferences: 
Not to be quoted without express written permission from the authors

$$
U=n_{t}^{\alpha} X_{t}^{\beta} Y_{t+1}^{1-\alpha-\beta}
$$

and production technology:

$$
f\left(k_{t}\right)=A_{t} k_{t}^{\rho}
$$

Thus, RC-improvement is achieved because the following relationships are satisfied:

1) if $\tilde{d}_{t}>\rho \alpha+(1-\rho)(1-\beta)$

$$
\delta_{t} / z<\Phi_{t} \equiv \min \left(1-\frac{\left(\tilde{d}_{t}-\alpha\right) \alpha}{\left(\tilde{d}_{t}-(1-\rho)(1-\beta)-\rho \alpha\right)\left(1-\beta-\tilde{d}_{t}\right)}, 1-\frac{\alpha}{1-\beta-\tilde{d}_{t}}\right)
$$

and

$$
d_{1} \equiv \rho \alpha+(1-\rho)(1-\beta)<\tilde{d}_{t}<d_{2} \equiv 1-\alpha-\beta
$$

2) if $\tilde{d}_{t}<\rho \alpha+(1-\rho)(1-\beta)$

$$
\begin{aligned}
& 1-\frac{\left(\alpha-\tilde{d}_{t}\right) \alpha}{\left((1-\rho)(1-\beta)+\rho \alpha-\tilde{d}_{t}\right)\left(1-\beta-\tilde{d}_{t}\right)}<\delta_{t} / z<1-\frac{\alpha}{1-\beta-\tilde{d}_{t}} \\
& \text { and } \\
& \tilde{d}_{t}<\min (\rho \alpha+(1-\rho)(1-\beta), 1-\alpha-\beta)
\end{aligned}
$$

See Appendix E for detailed calculations. Note that from (C-2) in Appendix C, this condition is also sufficient for CRC-improvement.

In this example, we quantify the RC-improvement in the real economy. Because the concern of the present paper is whether RC-improvement occurs by adjusting the amount of child benefit, 
Not to be quoted without express written permission from the authors

providing the parameters of the above function from the real economy allows us to derive the exact condition for RC-improvement.

In a typical national economy, the capital income ratio $\rho$ is 0.3 . Suppose that the preference $\alpha$ over children is set as 0.0025 or 0.005 and the preference $\beta$ over consumption during the young period is set as 0.6 or 0.7 . Thus, we can calculate the parameter $\Phi$ of equation (29) in Table 2. This parameter $\Phi$ is the upper limit of the child benefit amount under the constraint that $\tilde{d}_{t}$ satisfies equation (30). In other words, as long as the economy satisfies the condition $\delta / z<\Phi$, we can effect RC-improvement in the economy by increasing child benefit. In such a case, only the amount of debt matters, whereas fertility rate does not.

\section{Debt financing vs. tax financing}

In this section, we compare the advantages of financing child benefit programs using either public debt or lump-sum tax. To this end, we change the budget constraints of the government sector in our model as follows:

$$
\left\{\begin{array}{c}
d_{t}=\left(W_{t}-T_{t}\right) \tilde{d}_{t}+(1-\theta) \delta_{t} n_{t} \\
T_{t}=\frac{R_{t} d_{t-1}}{n_{t-1}}-d_{t}+\delta_{t} n_{t}
\end{array}\right.
$$

where $\theta$ stands for the ratio of tax resources to child-rearing support: if $\theta=1$, child benefit is financed by tax only, and if $\theta=0$, it is financed by public debt only. Then, from equations (21) and (31), we can derive the indirect utility function as follows:

$$
U=\left(W\left(\delta_{t}, \tilde{d}_{t-1}\right)-T\left(\delta_{t-1}, \delta_{t}, \tilde{d}_{t-1}, \tilde{d}_{t}, \theta\right)\right) \tilde{U}\left[\tilde{n}\left(\delta_{t}, \tilde{d}_{t-1}\right), \tilde{X}\left(\delta_{t}, \tilde{d}_{t-1}\right), \tilde{Y}\left(\delta_{t}, \tilde{d}_{t-1}\right)\right]
$$

where 
Not to be quoted without express written permission from the authors

$$
T\left(\delta_{t-1}, \delta_{t}, \tilde{d}_{t-1}, \tilde{d}_{t}, \theta\right) \equiv \frac{\frac{R_{t} \tilde{d}_{t-1}}{\tilde{n}_{t-1}}+R_{t}(1-\theta) \delta_{t-1}-W_{t} \tilde{d}_{t}+\theta \delta_{t} W_{t} \tilde{n}_{t}}{\left(1-\tilde{d}_{t}+\theta \delta_{t} \tilde{n}_{t}\right)}
$$

In this setting, we can derive the following proposition under the assumption of the preferences and production technology of Example 1.

Proposition 6 In the case of Example 1 with $\delta_{t-1}=\delta_{t}$ and $\tilde{d}_{t-1}=\tilde{d}_{t}$, when $\tilde{d}_{t}>d^{*}$, all tax financing $(\theta=1)$ is optimal. By contrast, when $\tilde{d}_{t}<d^{*}$, all debt financing $(\theta=0)$ is optimal if $0 \leq \delta_{t} \leq \delta^{*}$ and all tax financing $(\theta=1)$ is optimal if $\delta^{*}<\delta_{t}<z$, where $d^{*}$ and $\delta^{*}$ are denoted as follows:

$$
\begin{gathered}
d^{*} \equiv 1-\alpha-\beta-\frac{\rho}{1-\rho} \\
\delta^{*} \equiv\left[1-\frac{\alpha}{(1-\rho)(1-\alpha-\beta-\tilde{d})-(1-\alpha) \rho}\right] z
\end{gathered}
$$

Proof See Appendix F.

We have proven in Proposition 6 that debt financing is optimal if and only if $\tilde{d}_{t}<d^{*}$ and $\delta_{t}$ are sufficiently small. However, this proposition can be extended to a general utility function and a general production function.

Proposition 7 Under Assumptions 1, 2, and 3-2, namely weaker versions of the Inada condition, no child benefit condition, and stationary state condition, there exists $d^{*}$ such that debt 
financing is optimal if and only if $\tilde{d}<d^{*}$ holds, where:

1. Weaker version of Inada condition means $\lim _{k \rightarrow+0} f^{\prime}(k)>0$ and $\lim _{k \rightarrow \infty} f^{\prime}(k)=0$.

2. Stationary state means $\tilde{d}_{t} \equiv \tilde{d}, \tilde{n}_{t} \equiv \tilde{n}, \tilde{X}_{t} \equiv \tilde{X}, \tilde{Y}_{t} \equiv \tilde{Y}, k_{t} \equiv k, \delta_{t} \equiv \delta$.

3. No child benefit condition means $\delta \equiv 0$.

Proof See Appendix G.

We next return to Proposition 6, again using a Cobb-Douglas utility function and a specified production function. According to Proposition 6, debt financing is optimal only in the case of $\tilde{d}_{t}<d^{*}$. In addition, the condition that the sign of $\left(d^{*}+\delta \tilde{n}(\delta)\right)$ is positive is as follows:

$$
\begin{gathered}
1-\alpha-\beta-\frac{\rho}{1-\rho}+\frac{\alpha}{\mathrm{z} / \delta-1}>0 \\
\Leftarrow \quad \alpha<1-\beta-\frac{\rho}{1-\rho}
\end{gathered}
$$

In order to smooth consumption during young and old periods, we also assume the following:

Assumption 4 The preference parameters of Example 1 satisfy the following relationship:

$$
\begin{aligned}
& \beta>1-\alpha-\beta \\
& \Leftrightarrow \quad \alpha>1-2 \beta
\end{aligned}
$$

Then, from the constraint of $\alpha>0$, in equations (33) and (34), we can derive the following 
Not to be quoted without express written permission from the authors

corollary, as the necessary condition with regards to debt financing.

Corollary 1 Under Proposition 4 and Assumption 4, the necessary condition that debt financing is optimal is $\rho<1 / 3$.

Proof First, $\beta<1-\rho /(1-\rho)$ is derived from (33) and $\alpha>0$. Next, $\beta>\rho /(1-\rho)$ is derived from (33) and (34). Hence, from these relationships, $2 \rho /(1-\rho)<1$ holds.

Moreover, we can derive the following proposition and example in the case of tax financing, as shown in previous sections:

Proposition 8 RC-improvement is achieved by financing child benefit using lump-sum tax resources when the following is true:

Case 1: if $\eta_{\delta \delta}>\eta_{\delta n}$ and $\eta_{\delta s}>\frac{\delta_{t} n_{t}}{d_{t}}$

$$
n_{t}>\eta_{\delta R} \frac{1}{R_{t+1}} \frac{Y_{t+1}}{\delta_{t}} \text { and } \tilde{d}_{t-1}>\tilde{n}_{t-1} W_{t} / R_{t}
$$

Case 2: if $\tilde{s}_{\delta} \leq 0$ and $\tilde{n}_{\delta}>0$

$$
\eta_{\delta n}>\varepsilon_{\delta R}+\eta_{\delta W} \frac{\tilde{n}_{t-1} W_{t}}{R_{t} \tilde{d}_{t-1}} \text { and } \tilde{d}_{t-1}>\tilde{n}_{t-1} W_{t} / R_{t}
$$

Proof See Appendix H. 
Example 2 We assume the preferences and production technology that were described Example 1. Thus, the sufficient condition for RC-improvement in Proposition 8 is:

$$
\left\{\begin{array}{c}
\text { if } \frac{1-\beta}{1+\rho /(1-\rho)}<\tilde{d}_{t-1}<1-\alpha-\beta \\
\quad \delta_{t-1} / z<\min \left(1-\frac{\alpha\left[(2-\rho) \tilde{d}_{t-1}-(1-\rho)(1-\beta)\right]}{\left(1-\beta-\tilde{d}_{t-1}\right)\left[\tilde{d}_{t-1}-(1-\rho)(1-\beta)\right]}, 1-\frac{\alpha}{1-\beta-\tilde{d}_{t}}\right) \\
\text { if } \tilde{d}_{t-1}<\min \left(\frac{1-\beta}{1+\rho /(1-\rho)}, 1-\alpha-\beta\right) \\
\left.\quad 1-\frac{\alpha\left[(2-\rho) \tilde{d}_{t-1}-(1-\rho)(1-\beta)\right]}{\left(1-\beta-\tilde{d}_{t-1}\right)\left[\tilde{d}_{t-1}-(1-\rho)(1-\beta)\right.}\right]<\delta_{t-1} / z<1-\frac{\alpha}{1-\beta-\tilde{d}_{t}}
\end{array}\right.
$$

See Appendix I for detailed calculations.

\section{Conclusion}

In this paper, we derive the conditions for RC-efficiency in an endogenous population growth setting. Accordingly, when the elasticity of interest rates to child benefit is close to zero and there exists a huge amount of accumulated debt in the economy, financing child benefit programs by issuing debt and using lump-sum tax leads to RC-improvements.

The weakness of this study is that we make assumptions based on certain preferences, such as homogeneity. The findings would be more worthwhile if it were possible to show these results more generally. We will carry out this assignment in subsequent research. 
Not to be quoted without express written permission from the authors

Table 2. Range of Child Benefit with RC-improvement

1) Case 1: $\rho=0.3, \alpha=0.0025$ and $\beta=0.6$ or 0.7 .

\begin{tabular}{|c|c|c|c|c|c|c|}
\hline \multicolumn{3}{|c|}{ Preference Parameters of Utility } & \multicolumn{3}{|c|}{ Debt Parameters } & \multirow{2}{*}{$\begin{array}{c}\text { Upper Limit } \\
\text { of } \\
\text { Child Benefit } \\
\Phi\end{array}$} \\
\hline$\alpha$ & $\beta$ & $1-\alpha-\beta$ & $\tilde{d}$ & $d_{1}$ & $d_{1}$ & \\
\hline 0.0025 & 0.700 & 0.298 & 0.220 & 0.211 & 0.298 & 0.265 \\
\hline 0.0025 & 0.700 & 0.298 & 0.230 & 0.211 & 0.298 & 0.578 \\
\hline 0.0025 & 0.700 & 0.298 & 0.240 & 0.211 & 0.298 & 0.662 \\
\hline 0.0025 & 0.700 & 0.298 & 0.250 & 0.211 & 0.298 & 0.685 \\
\hline 0.0025 & 0.700 & 0.298 & 0.260 & 0.211 & 0.298 & 0.673 \\
\hline 0.0025 & 0.700 & 0.298 & 0.270 & 0.211 & 0.298 & 0.624 \\
\hline 0.0025 & 0.700 & 0.298 & 0.280 & 0.211 & 0.298 & 0.499 \\
\hline 0.0025 & 0.700 & 0.298 & 0.290 & 0.211 & 0.298 & 0.093 \\
\hline 0.0025 & 0.600 & 0.398 & 0.290 & 0.281 & 0.398 & 0.294 \\
\hline 0.0025 & 0.600 & 0.398 & 0.300 & 0.281 & 0.398 & 0.614 \\
\hline 0.0025 & 0.600 & 0.398 & 0.310 & 0.281 & 0.398 & 0.708 \\
\hline 0.0025 & 0.600 & 0.398 & 0.320 & 0.281 & 0.398 & 0.747 \\
\hline 0.0025 & 0.600 & 0.398 & 0.330 & 0.281 & 0.398 & 0.763 \\
\hline 0.0025 & 0.600 & 0.398 & 0.340 & 0.281 & 0.398 & 0.763 \\
\hline 0.0025 & 0.600 & 0.398 & 0.350 & 0.281 & 0.398 & 0.749 \\
\hline 0.0025 & 0.600 & 0.398 & 0.360 & 0.281 & 0.398 & 0.718 \\
\hline 0.0025 & 0.600 & 0.398 & 0.370 & 0.281 & 0.398 & 0.657 \\
\hline 0.0025 & 0.600 & 0.398 & 0.380 & 0.281 & 0.398 & 0.525 \\
\hline 0.0025 & 0.600 & 0.398 & 0.390 & 0.281 & 0.398 & 0.113 \\
\hline
\end{tabular}

2) Case 2: $\rho=0.3, \alpha=0.005$ and $\beta=0.6$ or 0.7 . 
Not to be quoted without express written permission from the authors

\begin{tabular}{|c|c|c|c|c|c|c|}
\hline \multicolumn{3}{|c|}{ Preference Parameters of Utility } & \multicolumn{3}{|c|}{ Debt Parameters } & \multirow{2}{*}{$\begin{array}{c}\text { Upper Limit } \\
\text { of } \\
\text { Child Benefit } \\
\Phi\end{array}$} \\
\hline$\alpha$ & $\beta$ & $1-\alpha-\beta$ & $\tilde{d}$ & $d_{1}$ & $d_{1}$ & \\
\hline 0.0050 & 0.700 & 0.295 & 0.230 & 0.212 & 0.295 & 0.131 \\
\hline 0.0050 & 0.700 & 0.295 & 0.240 & 0.212 & 0.295 & 0.313 \\
\hline 0.0050 & 0.700 & 0.295 & 0.250 & 0.212 & 0.295 & 0.364 \\
\hline 0.0050 & 0.700 & 0.295 & 0.260 & 0.212 & 0.295 & 0.343 \\
\hline 0.0050 & 0.700 & 0.295 & 0.270 & 0.212 & 0.295 & 0.245 \\
\hline 0.0050 & 0.600 & 0.395 & 0.300 & 0.282 & 0.395 & 0.203 \\
\hline 0.0050 & 0.600 & 0.395 & 0.310 & 0.282 & 0.395 & 0.405 \\
\hline 0.0050 & 0.600 & 0.395 & 0.320 & 0.282 & 0.395 & 0.489 \\
\hline 0.0050 & 0.600 & 0.395 & 0.330 & 0.282 & 0.395 & 0.521 \\
\hline 0.0050 & 0.600 & 0.395 & 0.340 & 0.282 & 0.395 & 0.523 \\
\hline 0.0050 & 0.600 & 0.395 & 0.350 & 0.282 & 0.395 & 0.496 \\
\hline 0.0050 & 0.600 & 0.395 & 0.360 & 0.282 & 0.395 & 0.435 \\
\hline 0.0050 & 0.600 & 0.395 & 0.370 & 0.282 & 0.395 & 0.313 \\
\hline 0.0050 & 0.600 & 0.395 & 0.380 & 0.282 & 0.395 & 0.048 \\
\hline
\end{tabular}


Not to be quoted without express written permission from the authors

\section{Appendix A: Proof of Proposition 2}

It is possible to show this using the same logic as that used by Michel and Wigniolle (2007). We follow their proof for the most part but change some points. In equilibrium, the market adjusts only the capital level, and thus we do not need to consider a change of $\delta$. Hence, for a given sequence of $\left\{\delta_{t}\right\}_{t=0}^{\infty}, \Gamma$ is represented in the following way:

$$
\Gamma(z-\bar{\delta}, k) \equiv \tilde{n}\left[z-\bar{\delta}, f^{\prime}(k)\right](k+\bar{\delta})-\tilde{s}\left[z-\bar{\delta}, f^{\prime}(k)\right]+\tilde{d}
$$

We show that $\Gamma=0$ has a unique solution. In order to show this, we check the property of $\Gamma$. First, we check the monotonicity of this function. The derivative of the first term $\left.\partial \tilde{n}\left[z-\bar{\delta}, f^{\prime}(k)\right](k+\bar{\delta})\right) / \partial k$ is positive, since $R=f^{\prime}(k)$ monotonically decreases in $k$ and $\eta_{R \tilde{n}} \leq \eta_{R k} \frac{k}{k+\delta} \Leftrightarrow \frac{R}{\tilde{n}} \frac{\partial \tilde{n}}{\partial R}<-\frac{R}{k} \frac{\partial k}{\partial R} \frac{k}{k+\delta} \Leftrightarrow \frac{\partial \tilde{n}}{\partial k}(k+\delta)+\tilde{n}>0$. The derivative of the second term $\partial \tilde{s}\left[z-\bar{\delta}, f^{\prime}(k)\right] / \partial k$ is negative, since $\tilde{s}_{R} \geq 0$. Hence, $\Gamma$ is increasing in $k$.

Next, we assume a certain level of child benefit $\delta$. At this time, when $k$ approaches 0 , it can be bounded in such a way that $k<1 \Rightarrow f^{\prime}(k)>f^{\prime}(1)$. Then, we obtain the following inequalities:

$$
\begin{aligned}
& \tilde{n}\left[z-\delta, f^{\prime}(k)\right] \leq \tilde{n}\left[z-\delta, f^{\prime}(1)\right] \\
& \tilde{s}\left[z-\delta, f^{\prime}(k)\right] \geq \tilde{s}\left[z-\delta, f^{\prime}(1)\right]
\end{aligned}
$$

and thus, 
Not to be quoted without express written permission from the authors

$$
\Gamma(z-\delta, k) \leq \tilde{n}\left[z-\delta, f^{\prime}(1)\right](k+\delta)-\tilde{s}\left[z-\delta, f^{\prime}(1)\right]+\tilde{d}
$$

Finally, we have

$$
\lim _{k \rightarrow 0} \Gamma(z-\delta, k) \leq \tilde{n}\left[z-\delta, f^{\prime}(1)\right] \delta-\tilde{s}\left[z-\delta, f^{\prime}(1)\right]+\tilde{d}=-\tilde{s}\left[z-\delta, f^{\prime}(1)\right]+\frac{d}{W-T}<0
$$

because $S_{t}>d_{t}$ must hold.

When $k$ approaches $+\infty$, we can prove the following using the contrary thought:

$k>1 \Rightarrow f^{\prime}(k)<f^{\prime}(1)$. We then obtain the following inequality:

$$
\Gamma(z-\delta, k) \geq \tilde{n}\left[z-\delta, f^{\prime}(1)\right](k+\delta)-\tilde{s}\left[z-\delta, f^{\prime}(1)\right]+\tilde{d}
$$

Thus:

$$
\lim _{k \rightarrow \infty} \Gamma(z-\delta, k)=+\infty
$$

Hence, for any given sequence $\forall\left\{\delta_{t}\right\}_{t=0}^{\infty}$, a unique inter-temporal equilibrium $\left\{k_{t+1}\right\}_{t=0}^{\infty}$ exists and the proposition has been proven. 
Not to be quoted without express written permission from the authors

\section{Appendix B: Proof of Proposition 3}

We use (25) in this proof. We first calculate the change in lifetime utility $\delta U_{t}$ when the amount of child benefit $\delta_{t}$ is increased:

$$
\begin{gathered}
\Delta U_{t}=\left(W_{t}-T_{t}\right)\left(\frac{\partial \tilde{U}_{t}}{\partial \tilde{n}_{t}} \frac{\partial \tilde{n}_{t}}{\partial \delta_{t}}+\frac{\partial \tilde{U}_{t}}{\partial \tilde{X}_{t}} \frac{\partial \tilde{X}_{t}}{\partial \delta_{t}}+\frac{\partial \tilde{U}_{t}}{\partial \tilde{Y}_{t+1}} \frac{\partial \tilde{Y}_{t+1}}{\partial \delta_{t}}\right) \Delta \delta_{t}+\tilde{U}_{t} \Delta\left(W_{t}-T_{t}\right) \\
\Delta U_{t}=\left(W_{t}-T_{t}\right)\left(\frac{\partial \tilde{U}_{t}}{\partial \tilde{n}_{t}} \frac{\partial \tilde{n}_{t}}{\partial \delta_{t}}+\frac{\partial \tilde{U}_{t}}{\partial \tilde{X}_{t}} \frac{\partial \tilde{X}_{t}}{\partial \delta_{t}}+\frac{\partial \tilde{U}_{t}}{\partial \tilde{Y}_{t+1}} \frac{\partial \tilde{Y}_{t+1}}{\partial \delta_{t}}\right) \Delta \delta_{t}+\tilde{U}_{t}\left(\frac{\partial W_{t}}{\partial \delta_{t-1}} \Delta \delta_{t-1}-\Delta T_{t}\right)
\end{gathered}
$$

It is possible to transform this equation using the household first-order conditions:

$$
\Delta U_{t}=\left(W_{t}-T_{t}\right) \lambda\left(\left(z-\delta_{t}\right) \frac{\partial \tilde{n}_{t}}{\partial \delta_{t}}+\frac{\partial \tilde{X}_{t}}{\partial \delta_{t}}+\frac{1}{R_{t+1}} \frac{\partial \tilde{Y}_{t+1}}{\partial \delta_{t}}\right) \Delta \delta_{t}+\tilde{U}_{t}\left(\frac{\partial W_{t}}{\partial \delta_{t-1}} \Delta \delta_{t-1}-\Delta T_{t}\right)
$$

Moreover, taking the derivative of equation (22) with respect to $\delta_{t}$, we obtain:

$$
\left(\left(z-\delta_{t}\right) \frac{\partial \tilde{n}_{t}}{\partial \delta_{t}}+\frac{\partial \tilde{X}_{t}}{\partial \delta_{t}}+\frac{1}{R_{t+1}} \frac{\partial \tilde{Y}_{t+1}}{\partial \delta_{t}}\right)=\tilde{n}_{t}+\frac{1}{R_{t+1}^{2}} \frac{\partial R_{t+1}}{\partial \delta_{t}} \tilde{Y}_{t}
$$

Then, by substituting this into the above equation, we have the following equation:

$$
\Delta U_{t}=\lambda\left(W_{t}-T_{t}\right)\left(\tilde{n}_{t}+\frac{1}{R_{t+1}^{2}} \frac{\partial R_{t+1}}{\partial \delta_{t}} \tilde{Y}_{t+1}\right) \Delta \delta_{t}+\tilde{U}_{t}\left(\frac{\partial W_{t}}{\partial \delta_{t-1}} \Delta \delta_{t-1}-\Delta T_{t}\right)
$$

where

$$
\Delta T_{t}=\frac{\partial T\left(\delta_{t-1}\right)}{\partial \delta_{t-1}} \Delta \delta_{t-1}=\frac{\partial\left(\frac{R_{t}}{\tilde{n}_{t-1}} \tilde{d}_{t-1}+R_{t} \delta_{t-1}-W_{t} \tilde{d}_{t}\right)}{\partial \delta_{t-1}} \frac{1}{1-\tilde{d}_{t}} \Delta \delta_{t-1}
$$

Using this, we can rewrite as:

$$
\Delta U_{t}=\lambda\left(W_{t}-T_{t}\right)\left(\tilde{n}_{t}+\frac{1}{R_{t+1}^{2}} \frac{\partial R_{t+1}}{\partial \delta_{t}} \tilde{Y}_{t+1}\right) \Delta \delta_{t}+\tilde{U}_{t}\left(\frac{\partial W_{t}}{\partial \delta_{t-1}}-\frac{\partial\left(\frac{R_{t}}{\tilde{n}_{t-1}} \tilde{d}_{t-1}+R_{t} \delta_{t-1}-W_{t} \tilde{d}_{t}\right)}{\partial \delta_{t-1}} \frac{1}{1-\tilde{d}_{t}}\right) \Delta \delta_{t-1}
$$


Not to be quoted without express written permission from the authors

This equation represents the effect of a change in child-rearing subsidies $\left\{\left(\Delta \delta_{t}\right)_{t=0}^{\infty}\right\}$ on the lifetime utility of generation $t$, given a set of debt policies $\left\{\left(d_{t}\right)_{t=0}^{\infty}\right\}$. If the sign of the big parentheses of the first term and the coefficient of the second term are both positive, it would be possible to offer welfare improvement to all generations by enlarging child benefit programs since the sign of $\tilde{U}_{t}$ is positive from homogeneity. Moreover, since $\partial W_{t} / \partial \delta_{t-1}>0$ from equations (15) and (25), the latter is always true in the case that the coefficient sign of the first term is positive. Namely, the sufficient condition of RC-improvement is as follows:

$$
n_{t}>\eta_{\delta R} \frac{1}{R_{t+1}} \frac{Y_{t+1}}{\delta_{t}}
$$

where

$$
\eta_{\delta R} \equiv-\frac{\delta_{t}}{R_{t+1}} \frac{\partial R_{t+1}}{\partial \delta_{t}}
$$

and

$$
\frac{\partial T_{t}}{\partial \delta_{t-1}}=\frac{\partial\left[R_{t} \tilde{n}_{t-1}\right.}{\left.\tilde{d}_{t-1}+R_{t} \delta_{t-1}-W_{t} \tilde{d}_{t}\right]} \frac{1}{1-\tilde{d}_{t}}<0
$$

This form (B-3) holds when:

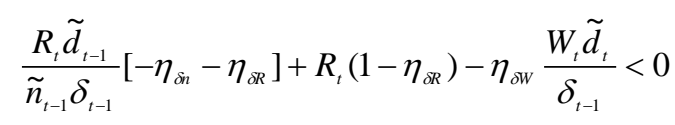

$$
\begin{aligned}
& \Leftrightarrow \quad-\eta_{\tilde{\delta}}-\eta_{\text {ฮR }}+\frac{\tilde{n}_{t} \delta_{t-1}}{\tilde{d}_{t-1}}\left(1-\eta_{\delta R}\right)-\eta_{\partial w} \frac{W_{t}}{R_{t}} \frac{\tilde{d}_{t} \tilde{n}_{t-1}}{\tilde{d}_{t-1}}<0
\end{aligned}
$$

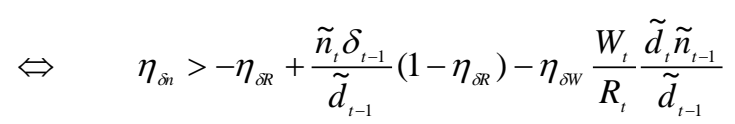

where 
Not to be quoted without express written permission from the authors

$$
\eta_{a v} \equiv \frac{\delta_{t}}{W_{t+1}} \frac{\partial W_{t+1}}{\partial \delta_{t}}
$$

Since $\eta_{\delta R}>0$ and $\eta_{\delta W}>0$, we can express this in the following form:

$$
\frac{\tilde{n}_{t-1} \delta_{t-1}}{\widetilde{d}_{t-1}}>-\eta_{\delta R}+\frac{\tilde{n}_{t} \delta_{t-1}}{\tilde{d}_{t-1}}\left(1-\eta_{\delta R}\right)-\eta_{\delta W} \frac{W_{t}}{R_{t}} \frac{\tilde{d}_{t} \tilde{n}_{t}}{\tilde{d}_{t-1}}
$$

As a result of (B-2), (B-4), and (B-5), we find the following sufficient conditions for RC-improvement:

$$
\begin{gathered}
n_{t}>\eta_{\delta R} \frac{1}{R_{t+1}} \frac{Y_{t+1}}{\delta_{t}} \\
\eta_{\delta n}>\frac{\tilde{n}_{t-1} \delta_{t-1}}{\tilde{d}_{t-1}}
\end{gathered}
$$

Let us briefly discuss CRC-improvement, which requires $\frac{d n_{t}}{d \delta_{t}}=\frac{d\left\{\tilde{n}_{t}\left(W_{t}-T_{t}\right)\right\}}{d \delta_{t}} \geq 0$ to hold additionally. $\frac{d\left(W_{t}-T_{t}\right)}{d \delta_{t}} \geq 0$ is already satisfied. Thus, $\frac{d \tilde{n}_{t}}{d \delta_{t}} \geq 0 \Leftrightarrow \frac{\partial \tilde{n}_{t}}{\partial \delta_{t}}+\frac{\partial k_{t}}{\partial \delta_{t}} \frac{\partial R_{t+1}}{\partial k_{t}} \frac{\partial \tilde{n}_{t}}{\partial R_{t+1}} \geq 0$ is the sufficient condition that the additional condition holds.

$$
\frac{\partial \tilde{n}_{t}}{\partial \delta_{t}} \geq 0 \text { holds from homogeneity, } \frac{\partial k_{t}}{\partial \delta_{t}} \geq 0 \text { holds by assumption, and } \frac{\partial R_{t+1}}{\partial k_{t}} \leq 0 \text { holds }
$$

according to Assumption 2. Thus, Assumption 3-2 is the sufficient condition that the additional condition holds. 
Not to be quoted without express written permission from the authors

\section{Appendix C: Proof of Proposition 4}

We use (25') in this proof. We first calculate the change in lifetime utility $\delta U_{t}$ when the amount of child benefit $\delta_{t}$ is increased. Thus, we can use the result obtained in the previous section:

$$
\Delta U_{t}=\lambda\left(W_{t}-T_{t}\right)\left(\tilde{n}_{t}+\frac{1}{R_{t+1}^{2}} \frac{\partial R_{t+1}}{\partial \delta_{t}} \tilde{Y}_{t+1}\right) \Delta \delta_{t}+\tilde{U}_{t}\left(\frac{\partial W_{t}}{\partial \delta_{t-1}} \Delta \delta_{t-1}-\Delta T_{t}\right)
$$

where

$$
\Delta T_{t}=\frac{\partial T\left(\delta_{t-1}\right)}{\partial \delta_{t-1}} \Delta \delta_{t-1}=\frac{\partial\left(\frac{R_{t}}{\tilde{n}_{t-1}} \tilde{d}_{t-1}+R_{t} \delta_{t-1}-W_{t} \tilde{d}_{t}\right)}{\partial \delta_{t-1}} \frac{1}{1-\tilde{d}_{t}} \Delta \delta_{t-1}
$$

Using $\Delta T_{t}$ in the original equation gives us:

$$
\Delta U_{t}=\lambda\left(W_{t}-T_{t}\right)\left(\tilde{n}_{t}+\frac{1}{R_{t+1}^{2}} \frac{\partial R_{t+1}}{\partial \delta_{t}} \tilde{Y}_{t+1}\right) \Delta \delta_{t}+\tilde{U}_{t}\left(\frac{\partial W_{t}}{\partial \delta_{t-1}}-\frac{\partial\left(\frac{R_{t}}{\tilde{n}_{t-1}} \tilde{d}_{t-1}+R_{t} \delta_{t-1}-W_{t} \tilde{d}_{t}\right)}{\partial \delta_{t-1}} \frac{1}{1-\tilde{d}_{t}}\right) \Delta \delta_{t-1}
$$

The first term is always positive in this case. We are interested in the sign of the second term.

To have sufficient conditions in order to improve the utility level, the term should be positive.

Hence:

$$
\frac{\partial W_{t}}{\partial \delta_{t-1}}-\frac{\partial\left(\frac{R_{t}}{\tilde{n}_{t-1}} \tilde{d}_{t-1}+R_{t} \delta_{t-1}-W_{t} \tilde{d}\right)}{\partial \delta_{t-1}} \frac{1}{1-\tilde{d}_{t}}>0
$$

The condition is thus calculated as:

$$
\frac{\partial W_{t}}{\partial \delta_{t-1}}-\left(\frac{\tilde{d}_{t-1}}{\tilde{n}_{t-1}} \frac{\partial R_{t}}{\partial \delta_{t-1}}-\frac{R_{t} \tilde{d}_{t-1}}{\tilde{n}_{t-1}^{2}} \frac{\partial \tilde{n}_{t-1}}{\partial \delta_{t-1}}+\frac{\partial R_{t}}{\partial \delta_{t-1}} \delta_{t-1}+R_{t}-\frac{\partial W_{t}}{\partial \delta_{t-1}} \tilde{d}_{t}\right) \frac{1}{1-\tilde{d}_{t}}>0
$$

Cancelling out the term $1 /\left(1-\tilde{d}_{t}\right)$, we can transform the condition as:

$$
\frac{\partial W_{t}}{\partial \delta_{t-1}}-\left(\frac{\tilde{d}_{t-1}}{\tilde{n}_{t-1}} \frac{\partial R_{t}}{\partial \delta_{t-1}}-\frac{R_{t} \tilde{d}_{t-1}}{\tilde{n}_{t-1}^{2}} \frac{\partial \tilde{n}_{t-1}}{\partial \delta_{t-1}}+\frac{\partial R_{t}}{\partial \delta_{t-1}} \delta_{t-1}+R_{t}\right)>0
$$


Not to be quoted without express written permission from the authors

$$
\begin{gathered}
\eta_{\delta W} \frac{W_{t}}{\delta_{t-1}}-\varepsilon_{\delta R} \frac{R_{t} \tilde{d}_{t-1}}{\tilde{n}_{t-1} \delta_{t-1}}+\frac{R_{t} \tilde{d}_{t-1}}{\tilde{n}_{t-1} \delta_{t-1}} \eta_{\delta n}-\varepsilon_{\delta R} R_{t}-R_{t}>0 \\
\eta_{\delta W} \frac{W_{t}}{\delta_{t-1}}+\frac{R_{t} \tilde{d}_{t-1}}{\tilde{n}_{t-1} \delta_{t-1}}\left(\eta_{\delta n}-\varepsilon_{\delta R}\right)-\left(1+\varepsilon_{\delta R}\right) R_{t}>0 \\
\eta_{\delta n}>\varepsilon_{\delta R}-\eta_{\delta W} \frac{W_{t} \tilde{n}_{t-1}}{R_{t} \tilde{d}_{t-1}}+\left(1+\varepsilon_{\delta R}\right) \frac{\tilde{n}_{t-1} \delta_{t-1}}{\tilde{d}_{t-1}}
\end{gathered}
$$

Hence, the sufficient condition is shown in the following:

$$
\eta_{\delta n}>\varepsilon_{\delta R}-\eta_{\delta W} \frac{W_{t} \tilde{n}_{t-1}}{R_{t} \tilde{d}_{t-1}}+\left(1+\varepsilon_{\delta R}\right) \frac{\tilde{n}_{t-1} \delta_{t-1}}{\tilde{d}_{t-1}}
$$

Let us briefly discuss CRC-improvement, which requires $\frac{d n_{t}}{d \delta_{t}}=\frac{d\left\{\tilde{n}_{t}\left(W_{t}-T_{t}\right)\right\}}{d \delta_{t}} \geq 0$ to hold additionally. $\frac{d\left(W_{t}-T_{t}\right)}{d \delta_{t}} \geq 0$ is already satisfied. Thus, $\frac{d \tilde{n}_{t}}{d \delta_{t}} \geq 0 \Leftrightarrow \frac{\partial \tilde{n}_{t}}{\partial \delta_{t}}+\frac{\partial k_{t}}{\partial \delta_{t}} \frac{\partial R_{t+1}}{\partial k_{t}} \frac{\partial \tilde{n}_{t}}{\partial R_{t+1}} \geq 0$ is the sufficient condition that the additional condition holds.

Note that $\frac{\partial \tilde{n}_{t}}{\partial \delta_{t}} \geq 0$ holds from homogeneity, $\frac{\partial k_{t}}{\partial \delta_{t}} \leq 0$ holds by assumption, and $\frac{\partial R_{t+1}}{\partial k_{t}} \leq 0$ holds by Assumption 2. Thus, $\frac{\partial \tilde{n}_{t}}{\partial R_{t+1}} \geq-\frac{\partial \tilde{n}_{t}}{\partial \delta_{t}} / \frac{\partial k_{t}}{\partial \delta_{t}} \frac{\partial R_{t+1}}{\partial k_{t}}$ is the sufficient condition that the additional condition holds.

In Example 1, it holds because $\frac{\partial \tilde{n}_{t}}{\partial R_{t+1}}=0$ holds. 


\section{Appendix D: Proof of Proposition 5}

This utility maximization problem is characterized as follows:

[Utility maximization] $\max U\left(\tilde{n}_{t}, \tilde{X}_{t}, \tilde{Y}_{t+1}\right)$ s.t. $\left(z-\delta_{t}\right) \tilde{n}_{t}+\tilde{X}_{t}+\frac{\tilde{Y}_{t+1}}{R_{t+1}}=1$

[Utility function] $U\left(\tilde{n}_{t}, \tilde{X}_{t}, \tilde{Y}_{t+1}\right)$ is homogeneous of degree one

[Assumption 3-2] $\frac{\partial \tilde{S}_{t}}{\partial R_{t}} \geq 0, \frac{\partial \tilde{n}_{t}}{\partial R_{t}} \leq 0$

[Assumption 2] $R_{t+1}=f^{\prime}\left(k_{t+1}\right)$

[Time evolution] $\tilde{n}_{t}\left(k_{t+1}+\delta_{t}\right)=\tilde{S}_{t}-\tilde{d}_{t}$ when $\tilde{S}_{t}=\frac{\tilde{Y}_{t+1}}{R_{t+1}}$

[Constancy] $\tilde{d}_{t}=$ const

Under this characterization, we generalize this maximization problem. First, let price vector be $\left(p_{1}, p_{2}, p_{3}\right)=\left(z-\delta_{t}, 1, \frac{1}{R_{t+1}}\right)$ and consumption vector be $\left(x_{1}, x_{2}, x_{3}\right)=\left(\tilde{n}_{t}, \tilde{X}_{t}, \tilde{Y}_{t+1}\right)$, where $z, d$ are constants. Then, the maximization problem is written as follows:

[Utility maximization] $\max U\left(x_{1}, x_{2}, x_{3}\right)$ s.t. $p_{1} x_{1}+p_{2} x_{2}+p_{3} x_{3}=1$

[Utility function] $U\left(x_{1}, x_{2}, x_{3}\right)$ is homogeneous of degree one

[Assumption 3-2] $\frac{\partial\left(p_{3} x_{3}\right)}{\partial p_{3}} \leq 0, \frac{\partial x_{1}}{\partial p_{3}} \geq 0$ 
[Assumption 2] $R=f^{\prime}(k), \quad p_{3}=\frac{1}{R}$

[Time evolution] $x_{1}\left(k-p_{1}+z\right)=p_{3} x_{3}-d$

Now that $p_{2}$ is constant, $x_{1}, x_{2}, x_{3}$ can be written as functions of $\left(p_{1}, p_{3}\right)$.

$$
\begin{aligned}
& x_{1}=x_{1}\left(p_{1}, p_{3}\right) \\
& x_{2}=x_{2}\left(p_{1}, p_{3}\right) \\
& x_{3}=x_{3}\left(p_{1}, p_{3}\right)
\end{aligned}
$$

Furthermore, because $U\left(x_{1}, x_{2}, x_{3}\right)$ is homogeneous of degree one, $\frac{\partial x_{1}}{\partial p_{3}}=\frac{\partial x_{3}}{\partial p_{1}}$ holds by Slutsky's theorem. Thus, from (D-9), the following holds:

$\frac{\partial x_{3}}{\partial p_{1}} \geq 0$

In addition, from the homogeneity of the utility function, the following holds:

$$
\frac{\partial x_{i}}{\partial p_{i}} \leq 0
$$

Assume $\frac{\partial k_{t+1}}{\partial \delta_{t}}>0$ now and we elicit a contradiction. From this assumption, the following holds:

$$
\frac{\partial k}{\partial p_{1}}<0
$$

As $f^{\prime \prime}(k)<0$, from (D-10), the following holds:

$$
\frac{\partial R}{\partial p_{1}}=\frac{\partial k}{\partial p_{1}} f^{\prime \prime}(k)>0
$$

From (D-10) and (D-16), the following holds: 
$\frac{\partial p_{3}}{\partial p_{1}}<0$

From (D-10) and the relationship between $k_{t+1}$ and $\delta_{t}$, the relationship between $p_{3}$ and $p_{1}$ is obtained. We express this relationship as $p_{3}=p_{3}\left(p_{1}\right)$ and the below as follows:

$x_{1}=x_{1}\left(p_{1}, p_{3}\left(p_{1}\right)\right)$

$x_{2}=x_{2}\left(p_{1}, p_{3}\left(p_{1}\right)\right)$

$x_{3}=x_{3}\left(p_{1}, p_{3}\left(p_{1}\right)\right)$.

$k=k\left(p_{1}\right)$

$R=R\left(p_{1}\right)$

(D-17) can be rewritten as:

$\frac{d p_{3}}{d p_{1}}<0 . \quad\left(\mathrm{D}-17^{\prime}\right)$

The relationship between total differentiation and partial differentiation can be expressed as follows:

$\frac{d x_{1}}{d p_{1}}=\frac{\partial x_{1}}{\partial p_{1}}+\frac{d p_{3}}{d p_{1}} \frac{\partial x_{1}}{\partial p_{3}}$

From (D-14), (D-17') and (D-9), the following is satisfied:

$\frac{d x_{1}}{d p_{1}}<0$

As $k_{t+1}$ only depends on $\delta$ (i.e., it only depends on $p_{1}$ ), (D-15) can be rewritten as follows:

$\frac{d k}{d p_{1}}<0$

(D-15’) 
The relationship between total differentiation and partial differentiation can also be expressed with (D-17'), (D-9), and (D-13):

$$
\begin{aligned}
& \frac{d\left(p_{3} x_{3}\right)}{d p_{1}}=\frac{d\left(p_{3}\left(p_{1}\right) x_{3}\left(p_{1}, p_{3}\left(p_{1}\right)\right)\right)}{d p_{1}} \\
& =\frac{d p_{3}\left(p_{1}\right)}{d p_{1}} x_{3}\left(p_{1}, p_{3}\left(p_{1}\right)\right)+p_{3}\left(p_{1}\right) \frac{d x_{3}\left(p_{1}, p_{3}\left(p_{1}\right)\right)}{d p_{1}} \\
& =\frac{d p_{3}\left(p_{1}\right)}{d p_{1}} x_{3}\left(p_{1}, p_{3}\left(p_{1}\right)\right)+p_{3}\left(p_{1}\right) \frac{\partial x_{3}\left(p_{1}, p_{3}\right)}{\partial p_{1}}+p_{3}\left(p_{1}\right) \frac{d p_{3}\left(p_{1}\right)}{d p_{1}} \frac{\partial x_{3}\left(p_{1}, p_{3}\right)}{\partial p_{3}} \\
& =\frac{d p_{3}\left(p_{1}\right)}{d p_{1}}\left(x_{3}\left(p_{1}, p_{3}\left(p_{1}\right)\right)+p_{3}\left(p_{1}\right) \frac{\partial x_{3}\left(p_{1}, p_{3}\right)}{\partial p_{3}}\right)+p_{3}\left(p_{1}\right) \frac{\partial x_{3}\left(p_{1}, p_{3}\right)}{\partial p_{1}} \\
& =\frac{d p_{3}\left(p_{1}\right)}{d p_{1}} \frac{\partial\left(p_{3} x_{3}\left(p_{1}, p_{3}\right)\right)}{\partial p_{3}}+p_{3}\left(p_{1}\right) \frac{\partial x_{3}\left(p_{1}, p_{3}\right)}{\partial p_{1}} \geq 0
\end{aligned}
$$

Here, we take the derivative of (D-11) by $p_{1}$. Then, the result is as follows:

$$
\frac{d x_{1}}{d p_{1}}\left(k-p_{1}+z\right)+x_{1}\left(\frac{d k}{d p_{1}}-1\right)=\frac{d\left(p_{3} x_{3}\right)}{d p_{1}}
$$

According to (D-20) and (D-15'), the left-hand side is negative. However, according to (D-21), the right-hand side is non-negative. This is a contradiction. 


\section{Appendix E: Calculation of Example 1}

The first-order conditions from household optimization are:

$$
\begin{gathered}
n_{t}=\frac{\alpha\left(W_{t}-T_{t}\right)}{z-\delta_{t}} \\
X_{t}=\beta\left(W_{t}-T_{t}\right) \\
Y_{t}=R_{t+1}(1-\alpha-\beta)\left(W_{t}-T_{t}\right) .
\end{gathered}
$$

We can normalize for $W_{t}-T_{t}$ :

$$
\begin{gathered}
\tilde{n}_{t}=\frac{\alpha}{z-\delta} \\
\tilde{X}_{t}=\beta \\
\tilde{Y}_{t}=R_{t+1}(1-\alpha-\beta) .
\end{gathered}
$$

Deriving the savings amount as:

$$
\begin{gathered}
S_{t}=(1-\alpha-\beta)\left(W_{t}-T_{t}\right) \\
\Rightarrow \quad \tilde{s}_{t}=(1-\alpha-\beta) .
\end{gathered}
$$

Factor prices are:

$$
\begin{gathered}
W_{t}=(1-\rho) A_{t} k_{t}^{\rho} \\
R_{t}=\rho A_{t} k_{t}^{\rho-1} .
\end{gathered}
$$

Obtaining the dynamics of $k$ :

$$
\begin{aligned}
& \frac{\alpha\left(k_{t+1}+\delta_{t}\right)}{z-\delta_{t}}=1-\alpha-\beta-\tilde{d}_{t} \\
\Leftrightarrow \quad & k_{t+1}=\frac{1-\alpha-\beta-\tilde{d}_{t}}{\alpha}\left(z-\delta_{t}\right)-\delta_{t}=\frac{1-\alpha-\beta-\tilde{d}_{t}}{\alpha} z-\frac{1-\beta-\tilde{d}_{t}}{\alpha} \delta_{t}
\end{aligned}
$$


Not to be quoted without express written permission from the authors

$$
\text { and } \tilde{d}_{t}<1-\alpha-\beta
$$

where $\quad k_{t+1}>0 \Leftrightarrow \quad \delta_{t} / z<1-\frac{\alpha}{1-\beta-\tilde{d}_{t}}$.

Therefore,

$$
\frac{\partial k_{t+1}}{\partial \delta_{t}}=-\frac{1-\beta-\tilde{d}_{t}}{\alpha}<0 .
$$

Calculate the elasticities:

$$
\begin{gathered}
\varepsilon_{\delta R}=\frac{\delta_{t}}{R_{t+1}} \frac{\partial R_{t+1}}{\partial \delta_{t}}=\frac{\delta_{t}}{A_{t+1} k_{t+1}^{\rho-1}} \frac{\partial\left(A_{t+1} k_{t+1}^{\rho-1}\right)}{\partial k_{t+1}} \frac{\partial k_{t+1}}{\partial \delta_{t}}=\frac{\delta_{t}}{k_{t+1}} \frac{(1-\rho)\left(1-\beta-\tilde{d}_{t}\right)}{\alpha}=\frac{(1-\rho) \delta_{t}}{\frac{1-\alpha-\beta-\tilde{d}_{t}}{1-\beta-\tilde{d}_{t}} Z-\delta_{t}} \\
\eta_{\delta W}=\frac{\delta_{t}}{W_{t+1}} \frac{\partial W_{t+1}}{\partial \delta_{t}}=\frac{\delta_{t}}{A_{t+1} k_{t+1}^{\rho}} \frac{\partial\left(A_{t+1} k_{t+1}^{\rho}\right)}{\partial k_{t+1}} \frac{\partial k_{t+1}}{\partial \delta_{t}}=-\frac{\delta_{t}}{k_{t+1}} \frac{\rho\left(1-\beta-\tilde{d}_{t}\right)}{\alpha}=-\frac{\rho \delta_{t}}{\frac{1-\alpha-\beta-\tilde{d}_{t}}{1-\beta-\tilde{d}_{t}} Z-\delta_{t}} \\
\eta_{\delta n}=\frac{\delta_{t}}{n_{t}} \alpha\left(W_{t}-T_{t}\right) \frac{\partial\left(1 /\left(z-\delta_{t}\right)\right)}{\delta_{t}}=\frac{\delta_{t}}{n_{t}} \frac{\alpha\left(W_{t}-T_{t}\right)}{\left(z-\delta_{t}\right)^{2}}=\frac{\delta_{t}}{Z-\delta_{t}} .
\end{gathered}
$$

We can derive sufficient conditions for RC-improvement:

$$
\begin{gathered}
\eta_{\delta n}>\varepsilon_{\delta R}-\eta_{\delta W} \frac{W_{t} \tilde{n}_{t-1}}{R_{t} \tilde{d}_{t-1}}+\left(1+\varepsilon_{\delta R}\right) \frac{\tilde{n}_{t-1} \delta_{t-1}}{\tilde{d}_{t-1}} \\
\Leftrightarrow \frac{\delta}{z-\delta}>\frac{(1-\rho) \delta}{\frac{1-\alpha-\beta-\tilde{d}}{1-\beta-\tilde{d}} z-\delta}+\frac{\rho \delta}{\frac{1-\alpha-\beta-\tilde{d}}{1-\beta-\tilde{d}} z-\delta} \frac{1-\rho}{\rho} \frac{1-\beta-\tilde{d}}{\alpha}\left(\frac{1-\alpha-\beta-\tilde{d}}{1-\beta-\tilde{d}} z-\delta\right) \frac{\tilde{n}}{\tilde{d}}+\left(1+\frac{(1-\rho) \delta}{\frac{1-\alpha-\beta-\tilde{d}}{1-\beta-\tilde{d}} z-\delta}\right) \frac{\tilde{n} \delta}{\tilde{d}} \\
\Leftrightarrow \frac{1}{z-\delta}>\frac{(1-\rho)}{\frac{1-\alpha-\beta-\tilde{d}}{1-\beta-\tilde{d}} z-\delta}+\frac{1-\rho}{1} \frac{1-\beta-\tilde{d}}{\alpha} \frac{\tilde{n}}{\tilde{d}}+\left(1+\frac{(1-\rho) \delta}{\frac{1-\alpha-\beta-\tilde{d}}{1-\beta-\tilde{d}} z-\delta}\right) \frac{\tilde{n}}{\tilde{d}}
\end{gathered}
$$


Not to be quoted without express written permission from the authors

$$
\begin{aligned}
& \Leftrightarrow \quad \frac{1}{z-\delta}>\frac{(1-\rho)}{z-\delta-\frac{\alpha}{1-\beta-\tilde{d}^{2}} z}+\frac{1-\rho}{1} \frac{1-\beta-\tilde{d}}{\alpha} \frac{\tilde{n}}{\tilde{d}}+\left(1+\frac{(1-\rho) \delta}{\left.z-\delta-\frac{\alpha}{1-\beta-\tilde{d}^{2}}\right)}\right) \frac{\tilde{n}}{\tilde{d}} \\
& \Leftrightarrow \quad \frac{z-\delta-\frac{\alpha}{1-\beta-\tilde{d}^{z}}>(1-\rho)+\frac{1-\rho}{1} \frac{1-\beta-\tilde{d}}{\alpha}\left(z-\delta-\frac{\alpha}{1-\beta-\tilde{d}} z\right) \frac{\tilde{n}}{\tilde{d}}+\left(z-\rho \delta-\frac{\alpha}{1-\beta-\tilde{d}} z\right) \frac{\tilde{n}}{\tilde{d}}}{\Leftrightarrow \quad\left(\rho(1-\beta-\tilde{d})-\frac{\alpha z}{z-\delta}\right) \frac{\tilde{d}}{\tilde{n}}>(1-\rho)(1-\beta-\tilde{d})\left((z-\delta) \frac{1-\beta-\tilde{d}}{\alpha}-z\right)+((z-\rho \delta)(1-\beta-\tilde{d})-\alpha z)}
\end{aligned}
$$

By using $Q \equiv 1-\beta-\tilde{d}, \quad \tilde{n}=\frac{\alpha}{z-\delta}$ and $(\mathrm{E}-1)$,

$$
\begin{aligned}
& \Leftrightarrow \quad \rho Q \frac{1-\beta-Q}{\tilde{n}}-\frac{\alpha z}{z-\delta} \frac{1-\beta-Q}{\tilde{n}}>(1-\rho) Q\left((z-\delta) \frac{Q}{\alpha}-z\right)+(z-\rho \delta) Q-\alpha z \\
& \Leftrightarrow \quad \rho Q \frac{1-\beta}{\tilde{n}}-\frac{\alpha z}{z-\delta} \frac{1-\beta-Q}{\tilde{n}}>\left(\frac{(1-\rho)(z-\delta)}{\alpha}+\frac{\rho}{\tilde{n}}\right) Q^{2}-(1-\rho) z Q+(z-\rho \delta) Q-\alpha z \\
& \Leftrightarrow \quad \frac{z-\delta}{\alpha} Q^{2}+\rho(z-\delta) Q-z Q-\rho Q(1-\beta) \frac{z-\delta}{\alpha}+(1-\alpha-\beta) z<0 \\
& \Leftrightarrow \quad \frac{z-\delta}{\alpha} Q^{2}-\left(\rho(z-\delta) \frac{1-\alpha-\beta}{\alpha}+z\right) Q+(1-\alpha-\beta) z<0 \\
& \Leftrightarrow \quad Q^{2}-\left(\rho(1-\alpha-\beta)+\frac{\alpha}{1-\delta / z}\right) Q+(1-\alpha-\beta) \frac{\alpha}{1-\delta / z}<0 \\
& \Leftrightarrow \quad(1-\alpha-\beta-Q) \frac{\alpha}{1-\delta / z}<(\rho(1-\alpha-\beta)-Q) Q \\
& \Leftrightarrow \quad(\tilde{d}-\alpha) \alpha<(1-\delta / z)(\tilde{d}-(1-\rho)(1-\beta)-\rho \alpha)(1-\beta-\tilde{d}) \\
& \Leftrightarrow\left\{\begin{array}{lll}
\delta / z<1-\frac{(\tilde{d}-\alpha) \alpha}{(\tilde{d}-(1-\rho)(1-\beta)-\rho \alpha)(1-\beta-\tilde{d})} & \text { and } & \tilde{d}>(1-\rho)(1-\beta)+\rho \alpha \\
\delta / z>1-\frac{(\tilde{d}-\alpha) \alpha}{(\tilde{d}-(1-\rho)(1-\beta)-\rho \alpha)(1-\beta-\tilde{d})} & \text { and } & \tilde{d}<(1-\rho)(1-\beta)+\rho \alpha
\end{array}\right.
\end{aligned}
$$

Therefore, from (E-1) to (E-3):

$$
\left\{\begin{array}{c}
\delta / z<\min \left(1-\frac{(\tilde{d}-\alpha) \alpha}{(\tilde{d}-(1-\rho)(1-\beta)-\rho \alpha)(1-\beta-\tilde{d})}, 1-\frac{\alpha}{1-\beta-\tilde{d}}\right) \\
\text { and } \rho \alpha+(1-\rho)(1-\beta)<\tilde{d}<1-\alpha-\beta \\
1-\frac{(\alpha-\tilde{d}) \alpha}{((1-\rho)(1-\beta)+\rho \alpha-\tilde{d})(1-\beta-\tilde{d})}<\delta / z<1-\frac{\alpha}{1-\beta-\tilde{d}} \\
\text { and } \tilde{d}<\min (1-\alpha-\beta, \rho \alpha+(1-\rho)(1-\beta))
\end{array}\right.
$$


Not to be quoted without express written permission from the authors

\section{Appendix F: Proof of Proposition 6}

In the indirect utility function (32), $T\left(\delta_{t-1}, \delta_{t}, \tilde{d}_{t-1}, \tilde{d}_{t}, \theta\right)$ depends on the parameter $\theta$. Hence, under the assumption that $\delta$ and $\tilde{d}$ are fixed, we can derive the maximum condition of (32) as follows:

$$
\min _{\theta} T\left(\delta_{t-1}, \delta_{t}, \tilde{d}_{t-1}, \tilde{d}_{t}, \theta\right)=\min _{\theta}\left[\frac{\frac{R_{t} \tilde{d}_{t-1}}{\tilde{n}_{t-1}}+R_{t}(1-\theta) \delta_{t-1}-W_{t} \tilde{d}_{t}+\theta \delta_{t} W_{t} \tilde{n}_{t}}{\left(1-\tilde{d}_{t}+\theta \delta_{t} \tilde{n}_{t}\right)}\right]
$$

To search for the optimal value $\theta$ of (F-1), we analyze the sign of the function $\partial T / \partial \theta$ using the preferences and production technology of Example 1.

The sign of $\partial T / \partial \theta=$ The sign of $\frac{\left.\partial \log \left[\frac{\frac{R_{t} \tilde{d}_{t-1}}{\tilde{n}_{t-1}+R_{t}(1-\theta) \delta_{t-1}-W_{t} \tilde{d}_{t}+\theta \delta_{t} W_{t} \tilde{n}_{t}}}{\left(1-\tilde{d}_{t}+\theta \delta_{t} \tilde{n}_{t}\right)}\right]\right)}{\partial \theta}$

$$
\begin{aligned}
& =\text { The sign of }\left[\frac{-R_{t} \delta_{t-1}+\delta_{t} W_{t} \tilde{n}_{t}}{\frac{R_{t} \tilde{d}_{t-1}}{\tilde{n}_{t}}+R_{t}(1-\theta) \delta_{t-1}-W_{t} \tilde{d}_{t}+\delta_{t} \theta W \tilde{n}_{t}}-\frac{\delta_{t} \tilde{n}_{t}}{\left(1-\tilde{d}_{t}+\theta \delta_{t} \tilde{n}_{t}\right)}\right] \\
& =\text { The sign of }\left[\left(1-\tilde{d}_{t}+\theta \delta_{t} \tilde{n}_{t}\right)\left(-R_{t} \delta_{t-1}+\delta_{t} W_{t} \tilde{n}_{t}\right)-\delta_{t} \tilde{n}_{t}\left(\frac{R_{t} \tilde{d}_{t-1}}{\tilde{n}_{t}}+R_{t}(1-\theta) \delta_{t-1}-W_{t} \tilde{d}_{t}+\delta_{t} \theta W \tilde{n}_{t}\right)\right] \\
& =\text { The sign of }\left[W_{t}-\left[\frac{\delta_{t-1}}{\delta_{t}}+\tilde{n}_{t} \delta_{t-1}+\tilde{d}_{t-1}-\tilde{d}_{t} \frac{\delta_{t-1}}{\delta_{t}}\right] R_{t} / \tilde{n}_{t}\right] \\
& =\text { The sign of } \Xi
\end{aligned}
$$


In addition, we can denote $\Xi$ as follows in the case with $\delta_{t-1}=\delta_{t}$ and $\tilde{d}_{t-1}=\tilde{d}_{t}$.

$$
\begin{aligned}
& \Xi=W_{t}-\left[\frac{1}{\tilde{n}_{t}}+\delta_{t-1}\right] R_{t} \quad(\mathrm{~F}-2) \\
& \left.\Leftrightarrow \quad \Xi(\delta)=(1-\rho) A k(\delta)^{\rho}-\frac{1}{\tilde{n}(\delta)}+\delta\right) \rho A k(\delta)^{\rho-1} \\
& \left.=(1-\rho) A\left[\frac{1-\alpha-\beta-\tilde{d}}{\alpha} z-\frac{1-\beta-\tilde{d}}{\alpha} \delta\right]^{\rho}-\frac{1}{\frac{\alpha}{z-\delta}}+\delta\right) \rho A\left[\frac{1-\alpha-\beta-\tilde{d}}{\alpha} z-\frac{1-\beta-\tilde{d}}{\alpha} \delta\right]^{\rho-1} \\
& =\{[(1-\rho)(1-\alpha-\beta-\tilde{d})-\rho] z-[(1-\rho)(1-\beta-\tilde{d})-(1-\alpha) \rho] \delta\} \times \frac{A}{\alpha}\left[\frac{1-\beta-\tilde{d}}{\alpha}\left(z^{*}-\delta\right)\right]^{\rho-1} \\
& \text { where } z^{*} \equiv \frac{1-\alpha-\beta-\tilde{d}}{1-\beta-\tilde{d}} z
\end{aligned}
$$

Because (F-2) does not depend on the parameter $\theta$ and $\lim _{\delta \rightarrow z^{*}-0} \Xi(\delta)=-\infty$, the sign of (F-2) is determined by the sign of the following value on (F-3):

$$
\Xi(0)=\{[(1-\rho)(1-\alpha-\beta-\tilde{d})-\rho] z\} \frac{A}{\alpha}\left[\frac{1-\alpha-\beta-\tilde{d}}{\alpha} z\right]^{\rho-1}
$$

The sign of (F-4) is subject to the following rules:

1) When $\tilde{d}>d^{*} \equiv 1-\alpha-\beta-\rho /(1-\rho), \quad \Xi(\delta)<0 \quad($ for $\quad \forall \delta \in[0, z) \quad$ ).

2) When $\tilde{d}<d^{*} \equiv 1-\alpha-\beta-\rho /(1-\rho)$, there exists $\delta *$ from $(\mathrm{F}-3)$, then $\Xi(\delta)>0 \quad\left(\quad\right.$ for $\quad \forall \delta \in\left[0, \delta^{*}\right) \quad$ ) or $\Xi(\delta)<0 \quad\left(\quad\right.$ for $\quad \forall \delta \in\left(\delta^{*}, z\right) \quad$ ) where $\delta *$ denotes as follows: 
Not to be quoted without express written permission from the authors

$$
\delta^{*} \equiv\left[1-\frac{\alpha}{(1-\rho)(1-\alpha-\beta-\tilde{d})-(1-\alpha) \rho}\right] z
$$

Therefore, from (F-2), (F-3), and the above rules, we can derive the following rules:

1) When $\tilde{d}>d^{*} \equiv 1-\alpha-\beta-\rho /(1-\rho), \quad \theta=1$ is optimal.

2) When $\tilde{d}<d^{*}, \quad \theta=0$ is optimal if $0 \leq \delta \leq \delta^{*}$ and $\theta=1$ is optimal if $\delta^{*}<\delta<z$. 


\section{Appendix G: Proof of Proposition 7}

Debt financing is optimal in the no child benefit condition if and only if $\Xi(0)>0$ holds if we use (F-2). Thus, what we would like to show is $\exists d^{*}$ s.t. $\tilde{d}<d^{*} \Leftrightarrow \Xi(0)>0$.

From (F-2), $\Xi\left(\delta_{t-1}\right)=W_{t}-\left[\frac{1}{\tilde{n}}+\delta_{t-1}\right] R_{t}$. Thus, under a stationary state condition, the following holds:

$\Xi(0)=W-\frac{R}{\tilde{n}}(\mathrm{G}-1)$

Thus, the following relationship holds.

$\Xi(0)>0 \Leftrightarrow W-\frac{R}{\tilde{n}}>0 \Leftrightarrow W>\frac{R}{\tilde{n}} \Leftrightarrow \frac{W}{R}>\frac{1}{\tilde{n}} \Leftrightarrow \frac{f(k)-k f^{\prime}(k)}{f^{\prime}(k)}>\frac{1}{\tilde{n}} \Leftrightarrow \frac{f(k)}{f^{\prime}(k)}-k>\frac{1}{\tilde{n}} \quad(\mathrm{G}-2)$

We can check these lemmas.

[Lemma G-1] $\frac{\partial}{\partial k} \frac{1}{\tilde{n}} \leq 0$

[Lemma G-2] $\frac{\partial}{\partial k}\left(\frac{f(k)}{f^{\prime}(k)}-k\right)>0$

[Lemma G-3] $\lim _{k \rightarrow+0} \frac{1}{\tilde{n}}>0$

[Lemma G-4] $\lim _{k \rightarrow+0}\left(\frac{f(k)}{f^{\prime}(k)}-k\right)=0$

[Lemma G-5] $\lim _{k \rightarrow \infty}\left(\frac{f(k)}{f^{\prime}(k)}-k\right)=\infty$ 
[Proof of Lemma G-1] Obvious from $\tilde{n}_{R} \leq 0 \Leftrightarrow \tilde{n}_{k} \geq 0$ in Assumption 3-2

[Proof of Lemma G-2] $\frac{\partial}{\partial k}\left(\frac{f(k)}{f^{\prime}(k)}-k\right)=\frac{\left(f^{\prime}(k)\right)^{2}-f(k) f^{\prime \prime}(k)}{\left(f^{\prime}(k)\right)^{2}}-1=-\frac{f(k) f^{\prime \prime}(k)}{\left(f^{\prime}(k)\right)^{2}}>0$

[Proof of Lemma G-3] Take arbitrary $k>0$. As $z>0$, $\tilde{n}<\infty$ holds. Thus, $\frac{1}{\tilde{n}}>0$. From Lemma G-1, $\frac{1}{\tilde{n}}$ weakly increases when $k$ approaches +0 . Thus $\lim _{k \rightarrow+0} \frac{1}{\tilde{n}}>0$ holds.

[Proof of Lemma G-4] Wage increases in $k ; \frac{\partial}{\partial k}\left(f(k)-k f^{\prime}(k)\right)=-k f^{\prime \prime}(k)>0$. In addition, interest rates decrease in $k ; \frac{\partial f^{\prime}(k)}{\partial k}=f^{\prime \prime}(k)<0$. Finally, $\lim _{k \rightarrow+0} f^{\prime}(k)>0$. Thus, when $k$ approaches $+0, \frac{f(k)-k f^{\prime}(k)}{f^{\prime}(k)}$ decreases and approaches zero.

[Proof of Lemma G-5] From $\frac{\partial}{\partial k}\left(f(k)-k f^{\prime}(k)\right)=-k f^{\prime \prime}(k)>0, \quad \frac{\partial f^{\prime}(k)}{\partial k}=f^{\prime \prime}(k)<0 \quad$ and $\lim _{k \rightarrow \infty} f^{\prime}(k)=0$, when $k$ approaches $\infty, \frac{f(k)-k f^{\prime}(k)}{f^{\prime}(k)}$ increases and approaches positive infinity.

From five lemmas, there exists $k^{*}>0$ such that $\frac{f(k)}{f^{\prime}(k)}-k>\frac{1}{\tilde{n}} \Leftrightarrow k>k^{*}$ holds. Also, from (G-2) and $\frac{f(k)}{f^{\prime}(k)}-k>\frac{1}{\tilde{n}} \Leftrightarrow k>k^{*}$, the following lemma holds.

[Lemma G-6] There exists $k^{*}>0$ such that $\Xi(0)>0 \Leftrightarrow k>k^{*}$ holds.

Last of all, we require the following lemma to hold. 
[Lemma G-7] Consider the following system.

$\max \tilde{U}(\tilde{n}, \tilde{X}, \tilde{Y})$ s.t. $z \tilde{n}+\tilde{X}+\frac{\tilde{Y}}{R}=1$ with following restrictions.

$\tilde{n} k=\tilde{S}-\tilde{d}, \quad \tilde{S}=\frac{\tilde{Y}}{R}$ and $R=f^{\prime}(k)$

Then, under Assumptions 1, 2, and 3-1 and the no child benefit condition (i.e., $\delta=0$ ), there is a bijection between $k$ and $\tilde{d}$ and the bijective function is a strictly decreasing function.

[Proof of Lemma G-7] $\tilde{n} k=\tilde{S}-\tilde{d} \Leftrightarrow \tilde{n} k-\tilde{S}=-\tilde{d}$. From Assumption 3-1, $\tilde{n} k$ is strictly increasing in $k$ and $\tilde{S}$ is weakly decreasing in $k$. Thus, $\tilde{n} k-\tilde{S}$ is strictly increasing in $k$. Thus, $\tilde{d}$ is strictly decreasing in $k . \tilde{n} k-\tilde{S}$ is a continuous function in $k$. Thus, $\tilde{n} k-\tilde{S}$ 's range is connected. Taking the connected area as $\tilde{d}$ 's domain, there is a continuous decreasing bijective function between $k$ and $\tilde{d}$.

From Lemma G-6 and Lemma G-7, the following holds.

There exists $d^{*}>0$ such that $\Xi(0)>0 \Leftrightarrow \tilde{d}<d^{*}$ holds. 
Not to be quoted without express written permission from the authors

\section{Appendix H: Proof of Proposition 8}

We use (25) and (25') in this proof. We first calculate the change in lifetime utility $\delta U_{t}$ when the amount of child benefit $\delta_{t}$ is increased. Thus, we can use (31) with $\theta=1$ and the results already obtained in the previous section:

$$
\Delta U_{t}=\lambda\left(W_{t}-T_{t}\right)\left(\tilde{n}_{t}+\frac{1}{R_{t+1}^{2}} \frac{\partial R_{t+1}}{\partial \delta_{t}} \tilde{Y}_{t+1}\right) \Delta \delta_{t}+\tilde{U}_{t}\left(\frac{\partial W_{t}}{\partial \delta_{t-1}} \Delta \delta_{t-1}-\Delta T_{t}\right)
$$

where

$$
\begin{aligned}
& \Delta T_{t}=\frac{\partial T\left(\delta_{t-1}, \delta_{t}\right)}{\partial \delta_{t-1}} \Delta \delta_{t-1}+\frac{\partial T\left(\delta_{t-1}, \delta_{t}\right)}{\partial \delta_{t}} \Delta \delta_{t}
\end{aligned}
$$

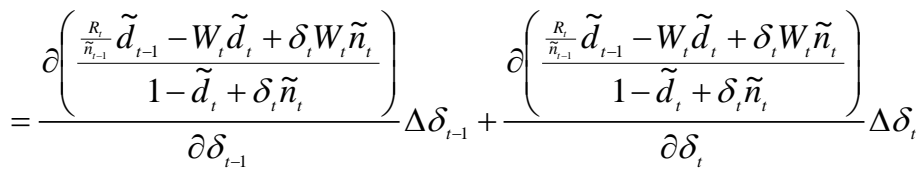

$$
\begin{aligned}
& =\frac{\partial\left(\frac{\frac{R_{t}}{\hat{n}_{t-1}} \tilde{d}_{t-1}-W_{t} \tilde{d}_{t}+\delta_{t} W_{t} \tilde{n}_{t}}{1-\tilde{d}_{t}+\delta_{t} \tilde{n}_{t}}\right)}{\partial \delta_{t-1}} \Delta \delta_{t-1}+\frac{\partial\left(W_{t}+\frac{\frac{R_{t}}{\tilde{n}_{t-1}} \tilde{d}_{t-1}-W_{t} \tilde{d}_{t}-W_{t}\left(1-\tilde{d}_{t}\right)}{1-\tilde{d}_{t}+\delta_{t} \tilde{n}_{t}}\right)}{\partial \delta_{t}} \Delta \delta_{t} \\
& =\frac{\partial\left(\frac{\frac{R_{t}}{\tilde{n}_{i-1}} \tilde{d}_{t-1}-W_{t} \tilde{d}_{t}+\delta_{t} W_{t} \tilde{n}_{t}}{1-\tilde{d}_{t}+\delta_{t} \tilde{n}_{t}}\right)}{\partial \delta_{t-1}} \Delta \delta_{t-1}-\frac{\frac{R_{t}}{\tilde{n}_{t-1}} \tilde{d}_{t-1}-W_{t}}{\left(1-\tilde{d}_{t}+\delta_{t} \tilde{n}_{t}\right)^{2}} \delta_{t} \tilde{n}_{t} \Delta \delta_{t}
\end{aligned}
$$

Using $\Delta T_{t}$ in the original equation:

$$
\begin{gathered}
\Delta U_{t}=\lambda\left(W_{t}-T_{t}\right)\left(\tilde{n}_{t}+\frac{1}{R_{t+1}^{2}} \frac{\partial R_{t+1}}{\partial \delta_{t}} \tilde{Y}_{t+1}\right) \Delta \delta_{t}+\tilde{U}_{t}\left(\frac{\partial W_{t}}{\partial \delta_{t-1}}-\frac{\partial\left(\frac{\frac{R_{i}}{\tilde{n}_{t-1}} \tilde{d}_{t-1}-W_{t} \tilde{d}_{t}+\delta_{t} W_{t} \tilde{n}_{t}}{1-\tilde{d}_{t}+\delta_{t} \tilde{n}_{t}}\right)}{\partial \delta_{t-1}}\right) \Delta \delta_{t-1} \\
+\tilde{U}_{t} \frac{\frac{R_{n}}{\tilde{n}_{t-1}} \tilde{d}_{t-1}-W_{t}}{\left(1-\tilde{d}_{t}+\delta_{t} \tilde{n}_{t}\right)^{2}} \delta_{t} \tilde{n}_{t} \Delta \delta_{t} \quad(\mathrm{H}-2)
\end{gathered}
$$


Case 1: if $\eta_{\dot{\delta}}>\eta_{\dot{\delta}}$ and $\eta_{\delta s}>\frac{\delta_{t} n_{t}}{d_{t}}$

In this case, we are interested in the signs of all the terms of $(\mathrm{H}-2)$. In order to have the sufficient conditions to improve the utility level, the terms should be positive. Hence:

$$
\tilde{n}_{t}+\frac{1}{R_{t+1}^{2}} \frac{\partial R_{t+1}}{\partial \delta_{t}} \tilde{Y}_{t+1}>0, \quad \frac{\partial W_{t}}{\partial \delta_{t-1}}-\frac{\partial\left(\frac{\frac{R_{t}}{\tilde{n}_{t-1}} \tilde{d}_{t-1}-W_{t} \tilde{d}_{t}+\delta_{t} W_{t} \tilde{n}_{t}}{1-\tilde{d}_{t}+\delta_{t} \tilde{n}_{t}}\right)}{\partial \delta_{t-1}}>0 .
$$

and

$$
\frac{R_{t}}{\tilde{n}_{t-1}} \tilde{d}_{t-1}-W_{t}>0
$$

The condition is calculated as follows:

$$
\tilde{n}_{t}+\frac{1}{R_{t+1}^{2}} \frac{\partial R_{t+1}}{\partial \delta_{t}} \tilde{Y}_{t+1}>0, \quad \frac{\partial W_{t}}{\partial \delta_{t-1}}-\frac{-\frac{R_{t}}{\tilde{n}_{t-1}^{2}} \frac{\partial \tilde{n}_{t-1}}{\partial \delta_{t-1}} \tilde{d}_{t-1}+\frac{1}{\tilde{n}_{t-1}} \frac{\partial R_{t}}{\partial \delta_{t-1}} \tilde{d}_{t-1}-\frac{\partial W_{t}}{\partial \delta_{t-1}} \tilde{d}_{t}+\delta_{t} \frac{\partial W_{t}}{\partial \delta_{t-1}} \tilde{n}_{t}}{1-\tilde{d}_{t}+\delta_{t} \tilde{n}_{t}}>0
$$

and

$$
\frac{R_{t}}{\tilde{n}_{t-1}} \tilde{d}_{t-1}-W_{t}>0
$$

Cancelling out the term $1 /\left(1-\tilde{d}_{t}\right)$, we can transform the condition as:

$$
n_{t}>\eta_{\delta R} \frac{1}{R_{t+1}} \frac{Y_{t+1}}{\delta_{t}}, \quad-\eta_{\delta W} \frac{W_{t}}{\delta_{t-1}}+\frac{R_{t} \tilde{d}_{t-1}}{\tilde{n}_{t-1} \delta_{t-1}} \eta_{\delta \emptyset}+\eta_{\delta R} \frac{R_{t} \tilde{d}_{t-1}}{\tilde{n}_{t-1} \delta_{t-1}}>0
$$

and 
Not to be quoted without express written permission from the authors

$$
\frac{R_{t}}{\widetilde{n}_{t-1}} \tilde{d}_{t-1}-W_{t}>0
$$

Since $\eta_{\delta R}>0$ and $\eta_{\delta w}<0$, the sufficient condition is shown as follows:

$$
n_{t}>\eta_{\delta R} \frac{1}{R_{t+1}} \frac{Y_{t+1}}{\delta_{t}}
$$

and

$$
\frac{R_{t}}{\tilde{n}_{t-1}} \tilde{d}_{t-1}>W_{t}
$$

Case 2: if $\tilde{s}_{\delta} \leq 0$ and $\tilde{n}_{\delta}>0$

In this case, the first term of $(\mathrm{H}-2)$ is always positive. We are interested only in the signs of the second and third terms of (H-2). Hence, from (H-3), the sufficient condition is shown as:

$$
\eta_{\delta n}>\varepsilon_{\delta R}+\eta_{\delta W} \frac{\tilde{n}_{t-1} W_{t}}{R_{t} \tilde{d}_{t-1}}
$$

and

$$
\frac{R_{t}}{\tilde{n}_{t-1}} \tilde{d}_{t-1}>W_{t}
$$


Not to be quoted without express written permission from the authors

\section{Appendix I: Proof of Example 2}

It is possible to transform (36) using the equations shown in Appendix D:

$$
\begin{aligned}
& \eta_{\tilde{\delta}}>\varepsilon_{\delta R}+\eta_{\delta W} \frac{\tilde{n}_{t-1} W_{t}}{R_{t} \widetilde{d}_{t-1}} \text { and } \frac{R_{t}}{\tilde{n}_{t-1}} \tilde{d}_{t-1}>W_{t} \\
& \Leftrightarrow \quad \frac{\delta_{t-1}}{z-\delta_{t}}>\frac{(1-\rho) \delta_{t-1}}{\frac{1-\alpha-\beta-\tilde{d}_{t-1}}{1-\beta-\tilde{d}_{t-1}} z-\delta_{t-1}}+\frac{\rho \delta_{t-1}}{\widetilde{d}_{t-1}} \frac{\alpha}{\left(z-\delta_{t-1}\right)} \frac{1-\rho}{\rho} \frac{1-\beta-\tilde{d}_{t-1}}{\alpha} \\
& \text { and } \\
& 1>\frac{1}{\tilde{d}_{t-1}} \frac{\alpha}{\left(z-\delta_{t-1}\right)} \frac{1-\rho}{\rho} \frac{1-\beta-\tilde{d}_{t-1}}{\alpha}\left[\frac{1-\alpha-\beta-\tilde{d}_{t-1}}{1-\beta-\tilde{d}_{t-1}} z-\delta_{t-1}\right] \\
& \Leftrightarrow \quad 1>\frac{(1-\rho)}{1-\frac{\alpha}{1-\beta-\tilde{d}_{t-1}} \frac{1}{1-\delta_{t-1} / z}}+\frac{(1-\rho)\left(1-\beta-\tilde{d}_{t-1}\right)}{\tilde{d}_{t-1}} \\
& \text { and } \\
& 1>\frac{(1-\rho)\left(1-\beta-\tilde{d}_{t-1}\right)}{\rho \tilde{d}_{t-1}}\left[1-\frac{\alpha}{1-\beta-\tilde{d}_{t-1}} \frac{1}{1-\delta_{t-1} / z}\right] \\
& \Leftrightarrow \quad \rho-\frac{\alpha}{1-\beta-\tilde{d}_{t-1}} \frac{1}{1-\delta_{t-1} / z}>\frac{(1-\rho)\left(1-\beta-\tilde{d}_{t-1}\right)}{\tilde{d}_{t-1}}\left[1-\frac{\alpha}{1-\beta-\tilde{d}_{t-1}} \frac{1}{1-\delta_{t-1} / z}\right] \\
& \text { and } \\
& \rho>\frac{(1-\rho)\left(1-\beta-\tilde{d}_{t-1}\right)}{\tilde{d}_{t-1}}\left[1-\frac{\alpha}{1-\beta-\tilde{d}_{t-1}} \frac{1}{1-\delta_{t-1} / z}\right] \\
& \Leftrightarrow \quad \rho-\frac{(1-\rho)\left(1-\beta-\tilde{d}_{t-1}\right)}{\tilde{d}_{t-1}}>\left[\frac{1}{1-\beta-\tilde{d}_{t-1}}-\frac{1-\rho}{\tilde{d}_{t-1}}\right] \frac{\alpha}{1-\delta_{t-1} / z}
\end{aligned}
$$

By using (E-1) and (E-2): 
Not to be quoted without express written permission from the authors

1) if $\frac{1-\beta}{1+\rho /(1-\rho)}<\tilde{d}_{t-1}<1-\alpha-\beta$

$$
\delta_{t-1} / z<\min \left(1-\frac{\alpha\left[(2-\rho) \tilde{d}_{t-1}-(1-\rho)(1-\beta)\right]}{\left(1-\beta-\tilde{d}_{t-1}\right)\left[\tilde{d}_{t-1}-(1-\rho)(1-\beta)\right]}, 1-\frac{\alpha}{1-\beta-\tilde{d}_{t}}\right)
$$

2) if $\tilde{d}_{t-1}<\min \left(\frac{1-\beta}{1+\rho /(1-\rho)}, 1-\alpha-\beta\right)$

$$
1-\frac{\alpha\left[(2-\rho) \tilde{d}_{t-1}-(1-\rho)(1-\beta)\right]}{\left(1-\beta-\tilde{d}_{t-1}\right)\left[\tilde{d}_{t-1}-(1-\rho)(1-\beta)\right]}<\delta_{t-1} / z<1-\frac{\alpha}{1-\beta-\tilde{d}_{t}}
$$


Not to be quoted without express written permission from the authors

\section{References}

ABEL, A. B., N. G. MANKIW, L. H. SUMMERS, and R. J. ZECKHAUSER (1989) Assessing dynamic efficiency: theory and evidence, Review of Economics Studies, 56(1), 1-20.

BECKER, G. S. (1960) An economic analysis of fertility, In Demographic and economic change in developed countries, National Bureau of Economic Research Conference Series 11, 209-231.

BECKER, G. S., and R. J. BARRO (1988) A reformulation of the economic theory of fertility, Quarterly Journal of Economics, 103, 1-25.

CHAKRABARTI, R. (1999) Endogenous fertility and growth in a model with old age support, Economic Theory, 13, 393-416.

CONDE-RUIZ, J. I., and E. L. GIMENEZ (2002) Perez-Nievas, M.: Efficiency in an overlapping generations model with endogenous population, mimeo.

DIAMOND, P. A. (1965) National debt in a neoclassical growth model, American Economic Review, 55(5), 1126-1150.

ECKSTEIN, Z., and K. WOLPIN (1985) Endogenous fertility and optimal population size, Journal of Public Economics, 27, 93-106.

GOLOSOV, M., L. E. JONES, and M. TERTILT (2004) Efficiency with endogenous population growth, National Bureau of Economic Research, 10231.

MICHEL, P., and B. WIGNIOLLE (2007) On Efficient Child Making, Economic Theory, 31(2), 307-326.

NISHIMURA, K., and J. ZHANG (1992) Pay-as-you-go public pensions with endogenous fertility, Journal of Public Economics, 48, 239-258.

RAUT, L. K., and T. N. SRINIVASAN (1994) Dynamics of endogenous growth, Economic Theory 4, 777-790.

UNITED NATIONS (2006) Demographic Yearbook

WILLIS, R. (1973) A new approach to the economic theory of fertility behavior, Journal of Political Economy, 81, S14-S64. 